\title{
Regularity and Decay of Lattice Green's Functions ${ }^{\star}$
}

\author{
Tadeusz Bałaban \\ Department of Physics, Harvard University, Cambridge, MA 02138, USA
}

\begin{abstract}
In the paper we study a class of lattice, covariant Laplace operators with external gauge fields. We prove that these operators are positive and that their Green's functions decay exponentially. They also have regularity properties similar to continuous space Green's functions. All the bounds are uniform in the lattice spacing.
\end{abstract}

\section{Introduction. Formulations of Theorems}

In this paper we prove some properties of Green's functions for difference Laplace operators. They imply all the properties of Green's functions (propagators) and Gaussian actions used in the papers [1-3], thereby completing the proof of ultraviolet stability of (Higgs) $)_{2,3}$ models. But the range of applicability of these properties is much wider and a need of them appears in many mathematical problems of statistical physics and quantum field theory. In fact, the mathematical estimates have an intrinsic interest of their own, so we present them in a self contained paper.

The properties of Green's functions we are interested in are regularity properties and exponential decay. The difference Laplace operators are lattice approximations to second order elliptic differential operators, so the regularity properties of lattice Green's functions are similar to the properties of continuous space Green's functions. For example, if $G$ is a lattice Green's function, then $\|G f\|_{2}$, $\left\|\partial_{\mu} G f\right\|_{2},\left\|\partial_{\mu} \partial_{\nu} G f\right\|_{2} \leqq c\|f\|_{2}$, where $\partial_{\mu}$ is a difference derivative and $\|\cdot\|_{2}$ denotes $L^{2}$-norm on the lattice. Similar estimates hold for other norms, and we are especially interested in Hölder norms. Exponential decay is interpreted in physics as the existence of an "effective mass". In mathematical terms this means that there is a strictly positive lower bound for the inverse Green's operators. The simplest example is the operator $-\Delta+m^{2}$. A Green's function $C_{m^{2}}=\left(-\Delta+m^{2}\right)^{-1}$ for this operator, on continuous space and on a lattice, satisfies a bound of the form $\left|C_{m^{2}}(x, y)\right| \leqq O(1)|x-y|^{-d+2} e^{-m|x-y|}$. We are interested in proving similar bounds, or bounds derived from these, for more general operators.

* Supported in part by the National Science Foundation under Grant No. PHY82-03669 
Let us formulate some basic definitions. We consider operators on subsets of the lattice $\eta Z^{d}, \eta=L^{-k}$. Here $L$ is a small positive integer $>1$, e.g. $L=2$ or 3 , and $k$ is an arbitrary positive integer. Another common case is to consider operators on subsets of a torus $T_{\eta}$ which we identify with a rectangular parallelepiped in $\eta Z^{d}$ with periodic conditions. The lattice $\eta Z^{d}$ is divided into unit cubes called blocks and parametrized by points $y$ of the unit lattice $Z^{d}$ :

$$
B^{k}(y)=\Delta(y)=\left\{x \in \eta Z^{d}: y_{\mu} \leqq x_{\mu}<y_{\mu}+1, \mu=1, \ldots, d\right\}, \quad y \in Z^{d} .
$$

We consider also a second division of $\eta Z^{d}$ into cubes of size $M$ called big blocks. They are parametrized by points of the lattice $M Z^{d}$ and defined in the same way as unit blocks with $M$ instead of 1 in (1.1). Here $M$ is a large positive integer defined later in this paper. We consider subsets $\Omega$ which are unions of big blocks. In order to define the class of Laplace operators, the so-called covariant Laplace operators, we have to introduce a notion of vector fields. These are real-valued functions defined on pairs $\left\langle x, x^{\prime}\right\rangle$ of nearest neighbour points of the lattice, called bonds. We identify them with vector-valued functions defined on points of the lattice by the identity $A_{\left\langle x, x+\eta e_{\mu}\right\rangle}=A_{\mu}(x)$, where $e_{\mu}$ is a unit vector of $\mu^{\text {th }}$ axis. The operators we are going to define depend on $A$ through the function

$U(A)=e^{q e \eta A}, \quad q$ is an antisymmetric $N \times N$ matrix, where $e$ is a real parameter.

Now the covariant Laplace operator $-\Delta_{A, \Omega}^{\eta, N}$ on a domain $\Omega$ with Neumann boundary conditions on $\partial \Omega$ is given by the following quadratic form defined on functions $\phi: \Omega \rightarrow R^{N}$

$$
\left\langle\phi,\left(-\Delta_{A, \Omega}^{\eta, N}\right) \phi\right\rangle=\sum_{b \subset \Omega} \eta^{d}\left|\left(D_{A}^{\eta} \phi\right)(b)\right|^{2}=\sum_{b \subset \Omega} \eta^{d}\left|\eta^{-1}\left(U\left(A_{b}\right) \phi\left(b_{+}\right)-\phi\left(b_{-}\right)\right)\right|^{2},
$$

where the summation is over the set of all bonds $b=\left\langle b_{-}, b_{+}\right\rangle$with end-points $b_{-}, b_{+}$in $\Omega$.

Next let us define some projection operators in the space of functions $\phi$. At first we define a covariant averaging operator

$$
\left(Q_{k}(A) \phi\right)(y)=\sum_{x \in B^{k}(y)} \eta^{d} U\left(A\left(\Gamma_{y, x}^{(k)}\right)\right) \phi(x), \quad y \in Z^{d} .
$$

Here $\Gamma_{y, x}^{(k)}, x \in B^{k}(y)$, are oriented contours in $B^{k}(y)$ connecting the initial point $y$ with a final point $x$. For an arbitrary contour $\Gamma$ in the lattice $\eta Z^{d}$ (considered as a sum of bonds) we define $A(\Gamma)=\sum_{b \subset \Gamma} A_{b}$, where orientations of the bonds $b$ agree with orientation of the contour $\Gamma$. The projection operator $P_{k}(A)$ is given by

$$
P_{k}(A)=Q_{k}^{*}(A) Q_{k}(A) .
$$

Our fundamental Green's function is a kernel of the operator

$$
G_{k}(\Omega, A)=\left(-\Delta_{A, \Omega}^{\eta, N}+m^{2}+a P_{k}(A)\right)^{-1},
$$

where $m^{2} \geqq 0$ and $a$ is a positive constant close to 1 . We consider all these operators under the assumption that the vector field $A$ is regular on $\Omega$ in the sense that

$$
\left|\left(\partial_{\mu}^{\eta} A\right)(x)\right| \leqq c e^{\beta-1}, \quad x \in \Omega, \quad \mu=1, \ldots, d, \quad \beta>0,
$$


and $c$ is some universal constant. [Of course $\partial_{\mu}^{\eta}$ is a difference derivative defined by $\left.\left(\partial_{\mu}^{\eta} A\right)(x)=\eta^{-1}\left(A\left(x+\eta e_{\mu}\right)-A(x)\right) \cdot\right]$

The operator defining the Green's function (1.6) has a strictly positive lower bound. More exactly we prove that there exists a positive constant $\gamma_{0}$ such that for $e$ sufficiently small and for a regular vector field $A$

$$
-\Delta_{A, \Omega}^{\eta, N}+a P_{k}(A) \geqq \gamma_{0} I .
$$

The constant $\gamma_{0}$ is independent of the lattice spacing $\eta$, as well as of $\Omega$ and of $A$. This bound justifies the definition (1.6) and explains exponential decay properties.

Finally for an arbitrary pair of points $x, x^{\prime} \in \eta Z^{d}$, let us denote by $\Gamma_{x, x^{\prime}}$ a shortest contour connecting these points. Now we can formulate the fundamental theorem of the paper.

Theorem (Proposition 2.1 of [1]). For $\alpha<1$ there exist positive constants $\delta_{0}, c_{0}, R_{0}$ independent of $A, k, \Omega$ and depending on $d, M$ only, $c_{0}$ on $\alpha$ also, such that for $e$ sufficiently small and for an arbitrary function $f: \Omega \rightarrow R^{N}$, we have

$$
\begin{aligned}
& \frac{1}{\left|x-x^{\prime}\right|^{\alpha}}\left|U\left(A\left(\Gamma_{x, x^{\prime}}\right)\right)\left(D_{A, \mu}^{\eta} G_{k}(\Omega, A) f\right)\left(x^{\prime}\right)-\left(D_{A, \mu}^{\eta} G_{k}(\Omega, A) f\right)(x)\right| \\
& \quad \leqq c_{0} \exp \left(-\delta_{0} \operatorname{dist}\left(\left\{x, x^{\prime}\right\}, \operatorname{supp} f\right)\right)\|f\|_{\infty}
\end{aligned}
$$

for $x, x^{\prime} \in \Omega$, and satisfying the condition $\operatorname{dist}\left(\left\{x, x^{\prime}\right\}, \Omega^{c}\right) \geqq R_{0}$. Similarly

$$
\left|\left(D_{A, \mu}^{\eta} G_{k}(\Omega, A) f\right)(x)\right|, \mid\left(G_{k}(\Omega, A)(x) \mid \leqq c_{0} \exp \left(-\delta_{0} \operatorname{dist}(x, \operatorname{supp} f)\right)\|f\|_{\infty}\right.
$$

for $x \in \Omega$, dist $\left(x, \Omega^{c}\right) \geqq R_{0}$. If $\Omega \subset \Omega_{0}$, then for $\delta G_{k}\left(\Omega, \Omega_{0}, A\right)$ defined by the equality

$$
\delta G_{k}\left(\Omega, \Omega_{0}, A\right)=G_{k}(\Omega, A)-G_{k}\left(\Omega_{0}, A\right),
$$

we have the inequalities (1.5) and (1.6) (with the same restrictions on $x, x^{\prime}$ ) with the additional factor

$$
\exp \left(-\delta_{0} \operatorname{dist}\left(\operatorname{supp} f, \Omega^{c}\right)-\delta_{0} \operatorname{dist}\left(\operatorname{supp} f, \Omega^{c}\right)\right)
$$

on the right hand sides. For some simple sets $\Omega$, e.g. for rectangular parallelepipeds, the inequalities hold without any restrictions on the points $x, x^{\prime}$, i.e. for all $x, x^{\prime} \in \Omega$.

This theorem implies in particular the Proposition 2.3 of [1]. It concerns unit lattice Green's functions $C_{A}^{(k)}(\Omega, A)$ of [1]. Let us recall that for operators $X$ defined on the unit lattice functions we define the operator $\left.X\right|_{\Lambda}$ restricted to a subset $\Lambda$ by $\left.X\right|_{\Lambda}=\Lambda X \Lambda$, where $\Lambda$ also denotes the characteristic function of the set $\Lambda$. We have

$$
C_{\Lambda}^{(k)}(\Omega, A)=\left(\left.\left(\Delta^{(k)}(\Omega, A)+a L^{-2} P(A)\right)\right|_{\Lambda}\right)^{-1} .
$$

Here $\Delta^{(k)}(\Omega, A)$ is an operator of the effective Gaussian action after $k$ renormalization transformations and can be defined by

$$
\Delta^{(k)}(\Omega, A)=a_{k} I-a_{k}^{2} Q_{k}(A) G_{k}(\Omega, A) Q_{k}^{*}(A),
$$

where $a_{k}$ is a constant proportional to $a$. Properties of the propagators $C_{\Lambda}^{(k)}(\Omega, A)$ are described in 
Proposition 2.3 of [1]. There exist positive constants $\delta_{0}, c_{0}, \gamma_{0}, \gamma_{1}$ dependent on $d$ and $M$ only and such that for arbitrary $\Lambda \subset \Omega^{(k)}=\Omega \cap Z^{d}, \Lambda$ being a sum of big blocks and for e sufficiently small, we have

$$
\begin{gathered}
\gamma_{0} I \leqq \Delta^{(k)}(\Omega, A)+a L^{-2} P(A) \leqq \gamma_{1} I, \\
\left|C_{\Lambda}^{(k)}\left(\Omega, A ; x, x^{\prime}\right)\right| \leqq c_{0} \exp \left(-\delta_{0}\left|x-x^{\prime}\right|\right), \quad x, x^{\prime} \in \Lambda .
\end{gathered}
$$

In particular the above inequality holds for $C^{(k)}(\Omega, A)$. Putting

$$
\delta C_{\Lambda}^{(k)}(\Omega, A)=C_{\Lambda}^{(k)}(\Omega, A)-C^{(k)}(\Omega, A),
$$

we have also

$$
\left|\delta C_{\Lambda}^{(k)}\left(\Omega, A ; x, x^{\prime}\right)\right| \leqq c_{0} \exp \left(-\delta_{0}\left(\left|x-x^{\prime}\right|+\operatorname{dist}\left(x, \Lambda^{c}\right)+\operatorname{dist}\left(x^{\prime}, \Lambda^{c}\right)\right)\right), \quad x, x^{\prime} \in \Lambda .
$$

Finally, for $\Omega \subset \Omega_{0}$ and

$$
\delta C_{\Lambda}^{(k)}\left(\Omega, \Omega_{0}, A\right)=C_{\Lambda}^{(k)}(\Omega, A)-C_{\Lambda}^{(k)}\left(\Omega_{0}, A\right),
$$

we have

$$
\begin{aligned}
& \left|\delta C_{\Lambda}^{(k)}\left(\Omega, \Omega_{0}, A ; x, x^{\prime}\right)\right| \\
& \quad \leqq c_{0} \exp \left(-\delta_{0}\left(\left|x-x^{\prime}\right|+\operatorname{dist}\left(x, \Omega^{(k) c}\right)+\operatorname{dist}\left(x^{\prime}, \Omega^{(k) c}\right)\right)\right), \quad x, x^{\prime} \in \Lambda .
\end{aligned}
$$

The last theorem we have to prove is the bound from below for the operator $\Delta^{(k)}(\Omega, A)$.

Proposition 3.1' of [2]. Let $\Omega$ be a sum of unit blocks (i.e. $\Omega^{(k)}$ is an arbitrary subset of $Z^{d}$ ) and let $A$ satisfies the condition

$$
\left|\left(\partial_{\mu}^{\eta} A\right)(x)\right| \leqq O(1) p(e) \quad\left(p(e)=a_{0}\left(1+\log e^{-1}\right)^{p}\right),
$$

then there exists a positive constant $\gamma_{0}$ depending on $d$ only, such that for $e$ sufficiently small

$$
\begin{aligned}
\left\langle\phi, \Delta^{(k)}(\Omega, A) \phi\right\rangle \geqq & \gamma_{0}\left(\sum_{\left\langle x, x^{\prime}\right\rangle \subset \Omega^{(k)}}\left|U\left(A\left(\left\langle x, x^{\prime}\right\rangle\right)\right) \phi\left(x^{\prime}\right)-\phi(x)\right|^{2}+m^{2} \sum_{x \in \Omega^{(k)}}|\phi(x)|^{2}\right) \\
& -O(1) e^{2-\alpha} \sum_{x \in \Omega^{(k)}}|\phi(x)|^{2}
\end{aligned}
$$

for arbitrary $\alpha>0$ and a constant $O(1)$ depending on $\alpha$ and the other constants, but independent of $\Omega, k, A$, and for an arbitrary function $\phi$.

This theorem implies (3.29) of [2].

The theorems will be proven in Sects. $2-4$ in the order in which they were written above. The fifth section will be devoted to a general theorem concerning operators on the unit lattice $Z^{d}$. There we have abstracted some basic features of our method and we have proven a theorem which, if applied to operators (1.14), gives another proof of Proposition 2.3.

\section{Generalized Random Walk Representation. Proof of the Theorem}

The proof will be done in several steps; in each step a reduction to a simpler problem will be achieved. Let us notice only that it is sufficient to prove the 
Proposition for a function $f$ with support in a unit cube $\Delta_{0}=B^{k}\left(y_{0}\right), y_{0} \in \Omega^{(k)}$; the general formulation is obtained by taking the decomposition $f=\sum_{\Delta \subset \Omega} \Delta f$ and
summing the inequalities.

In the first step we will introduce a generalization of random walk representations of [4], [5]. We have assumed that $\Omega^{(k)}$ is a sum of large blocks, i.e. blocks of the size $M$ on the unit lattice, thus $\Omega$ is a sum of the corresponding large blocks of the size $M$ on $\eta$-lattice $T_{\eta}$. For each $j \in Z^{d}$, let us define the set

$\square_{j}=\Omega \cap\{$ a sum of large blocks for which the point $M j$ is one of the vertices\} .

Let us observe that if the point $M j$ is not a boundary point of $\Omega$, then $\square_{j}$ is a cube of the size $2 M$ and with center in $M j$. For $M j$ lying on the boundary the set $\square_{j}$ is a sum of several $(<2 d)$ large blocks.

Next let us define a partition of unity $\left\{h_{j}\right\}_{j \in Z^{d}}$ on $T_{\eta}$. For each $j \in Z^{d}$, we take $h_{j}(x)=\prod_{\mu=1}^{d} h_{j_{\mu}}\left(x_{\mu}\right)$, and functions $h_{j}(x), j \in Z$, of one real variable $x$ are defined as $h_{j}(x)=h\left(\frac{x}{M}-j\right), h \in C_{0}^{\infty}(]-\frac{2}{3}, \frac{2}{3}[), h(x)=1$ for $x \in\left[-\frac{1}{3}, \frac{1}{3}\right]$, and it is chosen in such a way that $\sum_{j \in Z} h_{j}^{2}=1$.

Let us define an operator $G_{0}$ by the formula

$$
G_{0}=\sum_{j} h_{j} G_{k}\left(\square_{j}, \tilde{A}_{j}\right) h_{j},
$$

where the configurations $\underset{\tilde{A}}{\tilde{A}}$ are constructed in the following way: if $\square_{j}$ intersects the boundary of $\Omega$, then $\tilde{A}_{j}=A$; if $\square_{j}$ is an interior cube of $\Omega$, then we take $\tilde{A}_{j}$ as equal to $A$ on the cube $\left\{x:|x-M j| \leqq \frac{3}{4} M\right\}$, and changing regularly to a constant function in a neighbourhood of a boundary of $\square_{j}$. For example using the regularity condition (1.4) we can write the configuration $A$ on $\square_{j}$ as $A=A_{0}+A^{\prime}$, where $A_{0}$ is a constant configuration, e.g. $A_{0}=A(M j)$, and $A^{\prime}$ is regular and small, i.e. $\left|A^{\prime}\right|,\left|\partial_{\mu}^{\eta} A^{\prime}\right| \leqq c^{\prime} e^{\beta-1}$, with $c^{\prime}$ depending on $c, d$, and $M$, more exactly $c^{\prime}=d M c$. We take a function $\theta \in C_{0}^{\infty}(]-1,1[), \theta=1$ on $\left[-\frac{3}{4}, \frac{3}{4}\right]$, and we define $\theta_{j}(x)$ $=\prod_{\mu=1}^{d} \theta\left(\frac{x_{\mu}}{M}-j_{\mu}\right), \tilde{A}_{j}=A_{0}+\theta_{j} A^{\prime}$. In the sequel we will use this definition of $\tilde{A}_{j}$. Of course it satisfies the regularity condition (1.4) with another constant $c$.

We will prove that $G_{0}$ is a very good approximation of the operator $G_{k}(\Omega, A)$. Let us calculate $\left(-\Delta_{A, \Omega}^{\eta, N}+m_{k}^{2}+a_{k} P_{k}\right) G_{0}$. We have

$$
\left(D_{A}^{\eta} h \phi\right)(b)=h\left(b_{+}\right)\left(D_{A}^{\eta} \phi\right)(b)+\left(\partial^{\eta} h\right)(b) \phi\left(b_{-}\right),
$$

where $h$ is a real valued function

$$
\begin{aligned}
\left(D_{A}^{\eta *} g \theta\right)(x)= & \sum_{\mu=1}^{d} \eta^{-1}\left(g\left(\left\langle x-\eta e_{\mu}, x\right\rangle\right)-g\left(\left\langle x, x+\eta e_{\mu}\right\rangle\right)\right) \theta\left(\left\langle x, x+\eta e_{\mu}\right\rangle\right) \\
& +\sum_{\mu=1}^{d} g\left(\left\langle x-\eta e_{\mu}, x\right\rangle\right) \eta^{-1}\left(U\left(A_{\left\langle x, x-\eta e_{\mu}\right\rangle}\right) \theta\left(\left\langle x-\eta e_{\mu}, x\right\rangle\right)\right. \\
& \left.-\theta\left(\left\langle x, x+\eta e_{\mu}\right\rangle\right)\right) \\
= & \sum_{\mu=1}^{d}\left(\partial_{\mu}^{\eta *} g\right)(x) \theta\left(\left\langle x, x+\eta e_{\mu}\right\rangle\right)+\sum_{\mu=1}^{d} g\left(\left\langle x-\eta e_{\mu}, x\right\rangle\right)\left(D_{A, \mu}^{\eta *} \theta\right)(x),
\end{aligned}
$$


where $g, \theta$ are defined on bonds, $g$ is real valued and $\theta$ is a vector valued function. We assume also that $A_{-b}=-A_{b}$ for an arbitrary bond $b$. These formulas imply $\left(-\Delta_{A, \Omega}^{\eta, N} h \phi\right)(x)=\left(-\Delta_{\Omega}^{\eta, N} h\right)(x) \phi(x)-\sum_{b \in s t(x), b \subset \Omega}\left(\partial^{\eta} h\right)(b)\left(D_{A}^{\eta} \phi\right)(b)+h(x)\left(-\Delta_{A, \Omega}^{\eta, N} \phi\right)(x)$.

Special care has to be taken in considering boundary terms. Let us remark now that

$$
\left(-\Delta_{A, \Omega}^{\eta, N}\right) h_{j} G\left(\square_{j}, \tilde{A}_{j}\right)=\left(-\Delta_{\tilde{A}_{j}, \square_{j}}^{\eta, N}\right) h_{j} G\left(\square_{j}, \tilde{A}\right),
$$

because the function $h_{j}$ can be $\neq 0$ only on the part of the boundary of $\square_{j}$ which is contained in the boundary of $\Omega$. From (2.5), (2.6) we have

$$
\begin{aligned}
\left(-\Delta_{A, \Omega}^{\eta, N} G_{0}\right)\left(x, x^{\prime}\right)= & \sum_{j}\left[h_{j}(x)\left(-\Delta_{\tilde{A}_{j}, \square_{j}}^{\eta_{,},} G_{k}\left(\square_{j}, \tilde{A}_{j}\right)\right)\left(x, x^{\prime}\right) h_{j}\left(x^{\prime}\right)\right. \\
& -\sum_{b \in s t(x)}\left(\partial^{\eta} h_{j}\right)(b)\left(D_{\tilde{A}_{j}}^{\eta} G_{k}\left(\square_{j}, \tilde{A}_{j}\right)\right)\left(b, x^{\prime}\right) h_{j}\left(x^{\prime}\right) \\
& \left.+\left(-\Delta^{\eta} h_{j}\right)(x) G_{k}\left(\square_{j}, \tilde{A}_{j} ; x, x^{\prime}\right) h_{j}\left(x^{\prime}\right)\right],
\end{aligned}
$$

where we have used the fact that the normal derivative of $h_{j}$ to the boundary of $\square_{j}$ is equal to 0 .

For the operator $P_{k}(A)$, we have

$$
\begin{aligned}
\left(P_{k}(A) h \phi\right)(x) & =h(x)\left(P_{k}(A) \phi\right)(x)+\sum_{x^{\prime} \in B^{k}\left(y^{k}(x)\right)} \eta^{d} U\left(A\left(\Gamma_{x, y^{k}(x), x^{\prime}}^{(k)}\right)\left(\partial^{\eta} h\right)\left(\Gamma_{x, y^{k}(x), x^{\prime}}^{(k)}\right) \phi\left(x^{\prime}\right)\right. \\
& =: h(x)\left(P_{k}(A) \phi\right)(x)+\left(R_{k}\left(A, \partial^{\eta} h\right) \phi\right)(x) .
\end{aligned}
$$

Thus we get finally

$$
\begin{aligned}
((- & \left.\left.\Delta_{A, \Omega}^{\eta, N}+m_{k}^{2}+P_{k}(A)\right) G_{0}\right)\left(x, x^{\prime}\right) \\
= & \sum_{j}\left[h_{j}(x)\left(\left(-\Delta_{\tilde{A}_{j}, \square}^{\eta, N}+m_{k}^{2}+P_{k}\left(\tilde{A}_{j}\right)\right) G_{k}\left(\square_{j}, \tilde{A}_{j}\right)\right)\left(x, x^{\prime}\right) h_{j}\left(x^{\prime}\right)\right. \\
& \quad-\sum_{b \in s t(x)}\left(\partial^{\eta} h_{j}\right)(b)\left(D_{\tilde{A}_{j}} G_{k}\left(\square_{j}, \tilde{A}_{j}\right)\right)\left(b, x^{\prime}\right) h_{j}\left(x^{\prime}\right) \\
& +\left(-\Delta^{\eta} h_{j}\right)(x) G_{k}\left(\square_{j}, \tilde{A}_{j} ; x, x^{\prime}\right) h_{j}\left(x^{\prime}\right) \\
& +\left(R_{k}\left(\tilde{A}_{j}, \partial^{\eta} h_{j}\right) G_{k}\left(\square_{j}, \tilde{A}_{j}\right)\right)\left(x, x^{\prime}\right) h_{j}\left(x^{\prime}\right) \\
= & \delta^{\eta}\left(x-x^{\prime}\right)-\sum_{j}\left[\sum_{b \in s t(x)}\left(\partial^{\eta} h_{j}\right)(b)\left(D_{\tilde{A}_{j}}^{\eta} G_{k}\left(\square_{j}, \tilde{A}_{j}\right)\right)\left(b, x^{\prime}\right) h_{j}\left(x^{\prime}\right)\right. \\
& \left.+\left(\Delta^{\eta} h_{j}\right)(x) G_{k}\left(\square_{j}, \tilde{A}_{j} ; x, x^{\prime}\right) h_{j}\left(x^{\prime}\right)-\left(R_{k}\left(\tilde{A}_{j}, \partial^{\eta} h_{j}\right) G_{k}\left(\square_{j}, \tilde{A}_{j}\right)\right)\left(x, x^{\prime}\right) h_{j}\left(x^{\prime}\right)\right] .
\end{aligned}
$$

Let us define the operators

$$
\begin{gathered}
\left(K_{j} \phi\right)(x)=\square_{j}(x)\left[\sum_{b \in s t(x)}\left(\partial^{\eta} h_{j}\right)(b)\left(D_{\tilde{A}_{j}}^{\eta} \phi\right)(b)+\left(\Delta^{\eta} h_{j}\right)(x) \phi(x)\right. \\
\left.-\sum_{x^{\prime} \in B^{k}\left(y^{k}(x)\right)} \eta^{d}\left(\partial^{\eta} h_{j}\right)\left(\Gamma_{x, y^{k}(x), x^{\prime}}^{(k)}\right) U\left(\tilde{A}_{j}\left(\Gamma_{x, y^{k}(x), x^{\prime}}^{(k)}\right)\right) \phi\left(x^{\prime}\right)\right], \\
R=\sum_{j} K_{j} G_{k}\left(\square_{j}, \tilde{A}_{j}\right) h_{j} .
\end{gathered}
$$


In the sequel we will see that $R$ is a small operator in reasonable norms because $\left|\partial^{\eta} h_{j}\right| \leqq O\left(M^{-1}\right),\left|\Delta^{\eta} h_{j}\right| \leqq O\left(M^{-2}\right)$, so we have the representations

$$
G_{k}(\Omega, A)=G_{0}(I-R)^{-1}=\sum_{n=0}^{\infty} G_{0} R^{n} .
$$

They can be written in a very convenient form of "random walk" representation. Let us consider a space of paths $\omega$, each path is a sequence of points $\omega=\left\{\omega_{0}, \omega_{1}, \ldots, \omega_{n}\right\}, \omega_{i} \in Z^{d}$, satisfying the following condition: the points $\omega_{i}, \omega_{i+1}$ are vertices of a unit cube of the lattice. It can be formulated also that $\omega_{i+1}$ is of the form $\omega_{i+1}=\omega_{i}+\sum_{\mu=1}^{d} \varepsilon_{\mu} e_{\mu}$, where $\varepsilon_{\mu}$ is one of the numbers $-1,0,1$. We can write

$$
G_{k}(\Omega, A)=\sum_{\omega} h_{\omega_{0}} G_{k}\left(\square_{\omega_{0}}, \tilde{A}_{\omega_{0}}\right) h_{\omega_{0}} K_{\omega_{1}} G_{k}\left(\square_{\omega_{1}}, \tilde{A}_{\omega_{1}}\right) h_{\omega_{1}} \ldots K_{\omega_{n}} G_{k}\left(\square_{\omega_{n}}, \tilde{A}_{\omega_{n}}\right) h_{\omega_{n}},
$$

and this representation follows from (2.12) and the obvious fact that

$$
G_{k}\left(\square, \tilde{A}_{j}\right) h_{j} K_{j^{\prime}} G_{k}\left(\square_{j^{\prime}}, \tilde{A}_{j^{\prime}}\right)=0,
$$

if $\left|j-j^{\prime}\right|=\max _{\mu}\left|j_{\mu}-j_{\mu}^{\prime}\right|>1$. It is a basic representation and all the statements of Proposition I.2.1 follow from it and from some properties of the propagators $G_{k}\left(\square_{j}, \tilde{A}_{j}\right)$. Let us now formulate these properties in separate lemmas. We will need Hölder norms :

$$
\begin{aligned}
\|f\|_{1, \alpha}= & \max \left\{\sup _{x}|f(x)|, \sup _{x, \mu}\left|\left(D_{A, \mu}^{\eta} f\right)(x)\right|, \sup _{x, x^{\prime}, \mu} \frac{1}{\left|x^{\prime}-x\right|^{\alpha}} \mid U\left(A\left(\Gamma_{x, x^{\prime}}\right)\right)\right. \\
& \left.\cdot\left(D_{A, \mu}^{\eta} f\right)\left(x^{\prime}\right)-\left(D_{A, \mu}^{\eta} f\right)(x) \mid\right\},
\end{aligned}
$$

where the suprema are taken on a domain of the function $f$. This norm will be applied to expressions depending on some vector field, e.g. to $G_{k}\left(\square_{j}, \tilde{A}_{j}\right)$, and it will be understood that the same vector field is in the norm (2.14) as in these expressions.

Lemma 2.1. Let a set $\square$ be an arbitrary sum of unit blocks, but such that it is a sum of at most few large blocks, and let $A$ be as in the theorem. Then for e sufficiently small we have

$$
\begin{gathered}
\left\|G_{k}(\square, A) f\right\|_{2}, \quad\left\|D_{A, \mu}^{\eta} G_{k}(\square, A) f\right\|_{2}, \quad\left\|G_{k}(\square, A) D_{A, \mu}^{\eta *} f\right\|_{2}, \\
\left\|D_{A, \mu}^{\eta} G_{k}(\square, A) D_{A, v}^{\eta *} f\right\|_{2} \leqq c_{2}\|f\|_{2} .
\end{gathered}
$$

Let us notice that Lemma 2.1 implies that the $L^{2}$-norm of the operator $R$ given by (2.11) is small for $M$ large enough, so the series in the representation (2.12) is convergent in this norm.

Lemma 2.2. Let a rectangular parallelepiped $\square$ be a sum of few large blocks (e.g., as in the case of the cubes $\square_{j}$ ), and let $\tilde{A}$ be a regular vector field configuration in the sense of Proposition I.2.1, constant in a neighbourhood of the boundary of $\square$. Then for e sufficiently small and $\alpha<1$, there exists a constant $c_{1}$ depending on $d, \alpha$ only, such that

$$
\left\|G_{k}(\square, \tilde{A}) f\right\|_{1, \alpha} \leqq c_{1}\|f\|_{\infty}
$$


and a constant $c_{2}$ depending on $d, p_{1}$, such that

$$
\left\|G_{k}(\square, \tilde{A}) f\right\|_{q}, \quad\left\|D_{\tilde{A}, \mu}^{\eta} G_{k}(\square, \tilde{A}) f\right\|_{q}, \quad\left\|G_{k}(\square, \tilde{A}) D_{\tilde{A}, \mu}^{\eta *} f\right\|_{q} \leqq c_{2}\|f\|_{p}
$$

for $1 \leqq p, q \leqq \infty$, satisfying the condition $\frac{1}{p}-\frac{1}{p_{1}} \leqq \frac{1}{q} \leqq \frac{1}{p}$ with $p_{1}>d$.

These two lemmas together with the representation (2.13) imply the theorem.

Let us prove the inequality (1.9). We take an arbitrary pair of points $x, x^{\prime} \in \Omega$ with distances to the boundary of $\Omega$ greater than $R_{0}$. If $\left|x^{\prime}-x\right|>1$, then this inequality is a simple consequence of the corresponding inequality for the derivative only, hence we can assume $\left|x^{\prime}-x\right| \leqq 1$. If any of the points $x, x^{\prime}$ belongs to supp $h_{j}$, then both belong to $\square_{j}$ and in the representation (2.13) for each term we have that either both points belong to $\square_{\omega_{1}}$, or none. Of course the first situation occurs for at most $2^{d}$ cubes $\square_{j}$. We restrict the summation in (2.13) to paths starting in the corresponding $j$ 's and we will prove the inequality (1.9) using the representation $(2.13)$ :

(the left hand side of (1.9))

$$
\leqq \sum_{\omega}^{\prime}\left\|h_{\omega_{0}} G_{k}\left(\square_{\omega_{0}}, \tilde{A}_{\omega_{0}}\right) h_{\omega_{0}} K_{\omega_{1}} G_{k}\left(\square_{\omega_{1}}, \tilde{A}_{\omega_{1}}\right) h_{\omega_{2}} \cdot \ldots \cdot K_{\omega_{n}} G\left(\square_{\omega_{n}}, \tilde{A}_{\omega_{n}}\right) h_{\omega_{n}} f\right\|_{1, \alpha},
$$

where $\omega_{0}$ and $\omega_{n}$ are each restricted to $2^{d}$ possible values of $j$ by the conditions $x, x^{\prime} \in \square_{\omega_{0}}$, supp $f \subset \square_{\omega_{n}}$. We do not know yet if the series on the right side of (2.18) is convergent, and it will be one of the consequences of our estimates. Let us now take a positive integer $n_{0}$, we will fix it later, and let us define $R_{0}$ as

$$
\left.R_{0}=\text { (diameter of } \bigcup_{\substack{\omega=\left(\omega_{0}, \ldots, \omega_{n_{0}}\right) \\ \omega_{0}=0}} \bigcup_{i=1}^{n_{0}} \square_{\omega_{i}}\right)+2 M .
$$

Here $\square_{j}$ denotes a cube with center $M j$ and size equal to $2 M$. From this definition it follows that $R_{0}$ depends on $M$ and $n_{0}$. We divide the sum in (2.18) into two subsums: one with $n \leqq n_{0}$ and the other with $n>n_{0}$. The first is finite and the condition $\operatorname{dist}\left(\left\{x, x^{\prime}\right\}, \Omega^{c}\right) \geqq R_{0}$, together with the definition (2.19) of $R_{0}$ imply that all $\square_{\omega_{2}}$ are cubes contained in $\Omega$. The same of course holds for the first $n_{0}$ elements of the sequences $\omega$ in the second sum. We estimate the first sum using Lemma 2.2 by

$$
\begin{aligned}
& \sum_{\omega: n \leqq n_{0}}^{\prime} c_{1}\left\|K_{\omega_{1}} G_{k}\left(\square_{\omega_{1}}, \tilde{A}_{\omega_{1}}\right) h_{\omega_{1}}\right\|_{\infty} \cdot \ldots \cdot\left\|K_{\omega_{n}} G_{k}\left(\square_{\omega_{n}}, \tilde{A}_{\omega_{n}}\right) h_{\omega_{n}}\right\|_{\infty}\|f\|_{\infty} \\
& \quad \leqq \sum_{\omega: n \leqq n_{0}}^{\prime} c_{1}\left(c_{2} O(1) M^{-1}\right)^{n}\|f\|_{\infty} .
\end{aligned}
$$

We apply Lemma 2.2 to the terms of the second sum also, more exactly we apply (2.17) with $\frac{1}{p_{1}}=\frac{1}{2 n_{0}}$ and we fix $n_{0}$ such that $p_{1}=2 n_{0}>d+1$, e.g. $n_{0}=d$. We estimate the second sum by

$$
\begin{aligned}
\sum_{\omega: n>n_{0}}^{\prime} c_{1}\left\|K_{\omega_{1}} G_{k}\left(\square_{\omega_{1}}, \tilde{A}_{\omega_{1}}\right) h_{\omega_{1}}\right\|_{\infty, p_{1}} \prod_{i=2}^{n_{0}}\left\|K_{\omega_{i}} G_{k}\left(\square_{\omega_{i}}, \tilde{A}_{\omega_{i}}\right) h_{\omega_{i}}\right\| \frac{p_{1}}{i-1}, \frac{p_{1}}{i} \\
\cdot \prod_{i=n_{0}+1}^{n}\left\|K_{\omega_{i}} G_{k}\left(\square_{\omega_{i}}, \tilde{A}_{\omega_{i}}\right) h_{\omega_{i}}\right\|_{2,2}\|f\|_{2} \leqq \sum_{\omega: n>n_{0}}^{\prime} c_{1}\left(c_{2} O(1) M^{-1}\right)^{n}\|f\|_{\infty} .
\end{aligned}
$$


Here $\|T\|_{q, p}$ denotes a norm of an operator $T: L^{p} \rightarrow L^{q}$. Let us remark that the basic reason for the restriction $\operatorname{dist}\left(\left\{x, x^{\prime}\right\}, \Omega^{c}\right) \geqq R_{0}$ is that we do not have the inequalities (2.16) for the norms other than $L^{2}$-norms if $\square_{j}$ is not a cube (or rectangular parallelepiped). The summation in (2.18) is restricted to paths $\omega$ satisfying $x, x^{\prime} \in \square_{\omega_{0}}$, supp $f \subset \square_{\omega_{n}}$, so the length $n$ satisfies

$$
n \geqq M^{-1} \operatorname{dist}\left(\left\{x, x^{\prime}\right\}, \operatorname{supp} f\right)-2 \text {. }
$$

There are at most $2^{d}\left(3^{d}\right)^{n-1} 2^{d}$ of such paths, so finally we get the inequality

$$
\begin{aligned}
& \text { (the left hand side of }(1.9)) \leqq \sum_{n} 2^{d} c_{1}\left(3^{d} c_{2} O(1) M^{-1}\right)^{n}\|f\|_{\infty} \\
& \quad \leqq \frac{2^{d+1}}{e^{2}} e^{-M^{-1} \operatorname{dist}(\{x, x\}, \operatorname{supp} f)}\|f\|_{\infty},
\end{aligned}
$$

where $n \geqq M^{-1} \operatorname{dist}\left(\left\{x, x^{\prime}\right\}\right.$, supp $\left.f\right)-2$, and if $M$ is fixed such that $3^{d} c_{2} O(1) M^{-1} \leqq e^{-1}$. Thus the inequality (1.9) is proved, similarly the inequalities (1.10).

To prove the corresponding inequalities for $\delta G_{k}\left(\Omega, \Omega_{0}, A\right)=G_{k}(\Omega, A)$ $-G_{k}\left(\Omega_{0}, A\right)$ with $\Omega \subset \Omega_{0}$, we take the representations (2.13) for both propagators. The terms with $\omega$ such that $\square_{\omega_{2}}$ are interior cubes of $\Omega$ are the same in both representations, so they cancel in the difference, and for $\delta G_{k}$ we get a representation similar to (2.13) with the additional restriction that at least one $\square_{\omega_{r}}$ intersects the boundary $\partial \Omega$. We estimate the terms of the representation as above and we get the first inequality in (2.22) with $2^{d+1}$ instead of $2^{d}$ and with the restriction

$$
\begin{aligned}
n & \geqq M^{-1} \sup _{x_{1} \in \Omega^{c}}\left(\operatorname{dist}\left(\left\{x, x^{\prime}\right\}, x_{1}\right)+\operatorname{dist}\left(x_{1}, \operatorname{supp} f\right)\right)-3 \\
& \geqq(2 M)^{-1}\left(\operatorname{dist}\left(\left\{x, x^{\prime}\right\}, \operatorname{supp} f\right)+\operatorname{dist}\left(\left\{x, x^{\prime}\right\}, \Omega^{c}\right)+\operatorname{dist}\left(\operatorname{supp} f, \Omega^{c}\right)\right)-3 .
\end{aligned}
$$

It implies all the inequalities we need. Finally let us notice that if $\Omega$ is a rectangular parallelepiped, then all $\square_{j}$ in the representation (2.13) are cubes and we can apply Lemma 2.2 to all operators in it, so the restriction $\operatorname{dist}\left(\left\{x, x^{\prime}\right\}, \Omega^{c}\right) \geqq R_{0}$ is unnecessary. Thus we have proved the theorem, or rather reduced it to Lemmas 2.1, 2.2 .

Proof of Lemma 2.1. The first part of the proof is the same for Lemma 2.2 and is based on the ideas of the proof of Lemma 2.4 in [2]. We can write the configuration $A$ as a sum $A_{0}+A^{\prime}$, where $A_{0}$ is a constant configuration, e.g. it is a value of $A$ at some point of $\square$, and $A^{\prime}$ is a small and regular configuration, i.e. we have

$$
A=A_{0}+A^{\prime}, \quad\left|A^{\prime}\right|,\left|\partial_{\mu}^{\eta} A^{\prime}\right| \leqq c^{\prime} e^{\beta-1},
$$

$c^{\prime}$ depends on $c$ and $M$. Now we expand the propagator $G_{k}(\square, A)$ with respect to $A^{\prime}$. Using formula I.3.16 we have

$$
\begin{aligned}
G_{k}^{1 / 2} & \left(\square, A_{0}\right)\left(-\Delta_{A, \square}^{\eta, N}+m_{k}^{2}+a_{k} P_{k}(A)\right) G_{k}^{1 / 2}\left(\square, A_{0}\right) \\
= & I-G_{k}^{1 / 2}\left(\square, A_{0}\right)\left[F_{1, k}\left(-A^{\prime}\right)^{*} D_{A_{0}}^{\eta}+D_{A_{0}}^{\eta *} F_{1, k}\left(-A^{\prime}\right)\right. \\
& -F_{1, k}\left(-A^{\prime}\right)^{*} F_{1, k}\left(-A^{\prime}\right)-a_{k} F_{2, k}\left(A^{\prime}, A_{0}\right)^{*} Q_{k}\left(A_{0}\right) \\
& \left.-a_{k} Q_{k}^{*}\left(A_{0}\right) F_{2, k}\left(A^{\prime}, A_{0}\right)-a_{k} F_{2, k}\left(A^{\prime}, A_{0}\right)^{*} F_{2, k}\left(A^{\prime}, A_{0}\right)\right] G_{k}^{1 / 2}\left(\square, A_{0}\right) \\
= & : I-G_{k}^{1 / 2}\left(\square, A_{0}\right) V_{k} G_{k}^{1 / 2}\left(\square, A_{0}\right),
\end{aligned}
$$


where $F_{1, k}(A)=\eta^{-1}(U(A)-1)$,

$$
\left(F_{2, k}\left(A^{\prime}, A_{0}\right) \phi\right)(y)=\sum_{x \in B^{k}(y)} \eta^{d} F_{1, k}^{\prime}\left(A^{\prime}\left(\Gamma_{y, x}^{(k)}\right)\right) U\left(A_{0}\left(\Gamma_{y, x}^{(k)}\right)\right) \phi(x)
$$

and $F_{1, k}^{\prime}(A)=U(A)-1$. Taking into account the bound (2.23) and the inequalities

$$
\left\|G_{k}^{1 / 2}\left(\square, A_{0}\right)\right\|_{2,2}, \quad\left\|D_{A_{0}} G_{k}^{1 / 2}\left(\square, A_{0}\right)\right\|_{2,2}, \quad\left\|G_{k}^{1 / 2}\left(\square, A_{0}\right) D_{A_{0}}^{*}\right\|_{2,2} \leqq c_{0},
$$

holding for some absolute constant $c_{0}$, we can easily prove that

$$
\left\|G_{k}^{1 / 2}\left(\square, A_{0}\right) V_{k} G_{k}^{1 / 2}\left(\square, A_{0}\right)\right\|_{2,2} \leqq O(1) e^{\beta},
$$

with a constant $O(1)$ depending on $M$ only. For $e$ sufficiently small, we get the representation

$$
G_{k}(\square, A)=G_{k}^{1 / 2}\left(\square, A_{0}\right)\left(I-G_{k}^{1 / 2}\left(\square, A_{0}\right) V_{k} G_{k}^{1 / 2}\left(\square, A_{0}\right)\right)^{-1} G_{k}^{1 / 2}\left(\square, A_{0}\right),
$$

and a similar representation for derivatives of $G_{k}(\square, A)$, decomposing them on the right hand side according to the formula $D_{A}^{\eta}=U\left(A^{\prime}\right) D_{A_{0}}^{\eta}+F_{1, k}\left(A^{\prime}\right)$. From these representations and (2.25) we get easily the inequalities (2.15). Thus we have to prove the bounds $(2.25)$. Using the same gauge transformation as in the proof of Lemma 2.4, we reduce them to the case $A_{0}=0$. Now these bounds are consequences of quadratic form considerations. At first let us notice that $\square$ is a sum of unit cubes $\Delta$ and we have

$$
\left\langle\phi,\left(-\Delta_{\square}^{\eta, N}+m_{k}^{2}+a_{k} P_{k}\right) \phi\right\rangle \geqq \sum_{\Delta \subset \square}\left\langle\phi,\left(-\Delta_{\Delta}^{\eta, N}+a_{k} P_{k}\right) \phi\right\rangle .
$$

The operator $-\Delta_{\Delta}^{\eta, N}$ is bounded from below by $\pi^{2}$ on a subspace of functions on $\Delta$ orthogonal to constant functions, which are its eigenvectors corresponding to eigenvalue 0 . The operator $P_{k}$ is an orthogonal projection on a subspace of constant functions, thus

$$
\left\langle\phi,\left(-\Delta_{\Delta}^{\eta, N}+a_{k} P_{k}\right) \phi\right\rangle \geqq \min \left\{\pi^{2}, a_{k}\right\}\|\phi\|_{L^{2}(\Delta)},
$$

and from this and (2.26) we get

$$
0<G_{k}(\square, 0) \leqq c_{0} I, \quad c_{0}^{-1}=\min \left\{\pi^{2}, a_{k}\right\}, \quad \text { hence } \quad\left\|G_{k}(\square, 0) f\right\|_{2} \leqq c_{0}\|f\|_{2} .
$$

Further we have

$$
\begin{aligned}
\left\|\partial_{\mu}^{\eta} G_{k}^{1 / 2}(\square, 0) f\right\|_{2}^{2} & =\left\langle f, G_{k}^{1 / 2}(\square, 0) \partial_{\mu}^{\eta^{*}} \partial_{\mu}^{\eta} G_{k}^{1 / 2}(\square, 0) f\right\rangle \\
& \leqq\left\langle f, G_{k}^{1 / 2}(\square, 0)\left(-\Delta_{\square}^{\eta, N}+m_{k}^{2}+a_{k} P_{k}\right) G_{k}^{1 / 2}(\square, 0) f\right\rangle=\|f\|_{2}^{2},
\end{aligned}
$$

hence $\left\|\partial_{\mu}^{\eta} G_{k}^{1 / 2}(\square, 0)\right\|_{2,2}=\left\|G_{k}^{1 / 2}(\square, 0) \partial_{\mu}^{\eta^{*}}\right\|_{2,2} \leqq 1$, and this together with (2.28) imply the bounds (2.25). Thus the Lemma 2.1 is proved.

Remark. Let us notice that this lemma alone implies a weaker version of Proposition I.2.1 with $L^{2}$-norms. More exactly we have

Corollary 2.3. If $\Omega$ and $A$ are as in Proposition I.2.1, then there exist positive constants $c_{0}, \delta_{0}$ such that for arbitrary scalar field configurations $f, f^{\prime}$ defined on $\Omega$, we have

$$
\begin{gathered}
\left|\left\langle f, G_{k}(\Omega, A) f^{\prime}\right\rangle\right|, \quad\left|\left\langle f, D_{A, \mu}^{\eta} G_{k}(\Omega, A) f^{\prime}\right\rangle\right|, \quad\left|\left\langle f, G_{k}(\Omega, A) D_{A, v}^{\eta^{*}} f^{\prime}\right\rangle\right|, \\
\left.K f, D_{A, \mu}^{\eta} G_{k}(\Omega, A) D_{A, v}^{\eta^{*}} f^{\prime}\right\rangle \mid \leqq c_{0} e^{-\delta_{0} \operatorname{dist}\left(\operatorname{supp} f, \operatorname{supp} f^{\prime}\right)}\|f\|_{2}\left\|f^{\prime}\right\|_{2} .
\end{gathered}
$$


The same inequalities hold for $\delta G_{k}\left(\Omega, \Omega_{0}, A\right)$ with the additional factor $e^{-\delta_{0}\left(\operatorname{dist}\left(\operatorname{supp} f, \Omega^{c}\right)+\operatorname{dist}\left(\operatorname{supp} f^{\prime}, \Omega^{c}\right)\right)}$.

Of course it is enough to prove it for $f, f^{\prime}$ with supports in unit cubes, and the proof proceeds as before using only the $L^{2}$-bounds of Lemma 2.1. Let us notice also that now there are no restrictions on supports of $f, f^{\prime}$, so in this aspect the Corollary is a little bit stronger than Proposition I.2.1.

Now we begin a

Proof of Lemma 2.2. It is much longer and will be reduced to some other lemmas again. We start with the same operations as in the proof of Lemma 2.2 and we have the representation (2.26) for $e$ sufficiently small, which we can write in the following form

$$
G_{k}(\square, \tilde{A})=\sum_{n=0}^{\infty} G_{k}\left(\square, A_{0}\right)\left(V_{k} G_{k}\left(\square, A_{0}\right)\right)^{n} .
$$

Now we have to choose $A_{0}$ more carefully. According to our assumptions $\tilde{A}$ is constant in a neighbourhood of boundary of $\square$, and we choose $A_{0}$ equal to this constant. Thus in the decomposition $\tilde{A}=A_{0}+A^{\prime}$, the configuration $A^{\prime}$ is regular and has a compact support in $\square$. The series in (2.31) is convergent in $L^{2}(\square)$, but we will prove that it is in fact convergent in stronger norms appearing in formulation of Lemma 2.2.

Let us assume that Lemma 2.2 holds for $G_{k}\left(\square, A_{0}\right)$ with constant configurations $A_{0}$. We will prove it for a general case using (2.31).

At first let us observe that $V_{k}$ can be interpreted as a first order differential operator acting on a function on the right hand side of it. The only trouble is with the term $D_{A_{0}}^{\eta *} F_{1, k}\left(-A^{\prime}\right)$, for which it is not a natural interpretation. Using the formula (2.4) and the fact that $A^{\prime}$ has a compact support in $\square$, we have

$$
\begin{aligned}
\left(D_{A_{0}}^{\eta *} F_{1, k}\left(-A^{\prime}\right) f\right)(x)= & \left(\partial^{\eta *} F_{1, k}\left(-A^{\prime}\right)\right)(x) f(x)+\sum_{\mu=1}^{d} F_{1, k}\left(-A_{\left\langle x-\eta e_{\mu}, x\right\rangle}^{\prime}\right)\left(D_{A_{0}, \mu}^{\eta *} f\right)(x) \\
= & -\sum_{\mu=1}^{d}\left(\partial_{\mu}^{\eta} F_{1, k}\left(-A_{\mu}^{\prime}\right)\right)\left(x-\eta e_{\mu}\right) f(x) \\
& -\sum_{\mu=1}^{d} F_{1, k}\left(-A_{\mu}^{\prime}\left(x-\eta e_{\mu}\right)\right) U\left(-A_{0, \mu}\right)\left(D_{A_{0}, \mu}^{\eta} f\right)\left(x-\eta e_{\mu}\right) .
\end{aligned}
$$

Thus it is a first order differential operator with small coefficients. Only here we needed the assumption that $\tilde{A}$ is constant in a neighbourhood of $\hat{\partial} \square$.

Now using Lemma 2.2 for $G_{k}\left(\square, A_{0}\right)$ and the decomposition $D_{\tilde{A}}^{\eta}=U\left(A^{\prime}\right) D_{A_{0}}^{\eta}$ $+F_{1, k}\left(A^{\prime}\right)$, we have

$$
\left\|G_{k}(\square, \tilde{A}) f\right\|_{1, \alpha} \leqq O(1) c_{1} \sum_{n=0}^{\infty}\left\|\left(V_{k} G_{k}\left(\square, A_{0}\right)\right)^{n} f\right\|_{\infty} \leqq O(1) c_{1} \sum_{n=0}^{\infty}\left(O(1) e^{\beta} c_{2}\right)^{n}\|f\|_{\infty} .
$$

The series on the right hand side is convergent for $e$ sufficiently small, and we get the inequality (2.16). The inequality (2.17) is proved in the same way.

Lemma 2.2 in the case of a constant configuration $A_{0}$ is equivalent to the case of configuration $A_{0}=0$ by the same argument with the gauge transformation as before. Thus we have reduced the proof of this lemma to a proof of the 
corresponding properties for the propagator $G_{k}(\square, 0)$, or to the one-component propagator $G_{k}(\square)\left[G_{k}(\square, 0)=G_{k}(\square) 1,1\right.$ is identity operator on $\left.R^{N}\right]$. This proof is based on renormalization group equations (2.43) of [1] rescaled to the $\eta$-lattice:

$$
G_{k}(\square)=C^{(0), \eta}(\square)+\sum_{j=1}^{k-1} a_{j}^{2}\left(L^{j} \eta\right)^{-4} G_{j}^{\eta}(\square) Q_{j}^{*} C^{(j), L \jmath}(\square) Q_{j} G_{j}^{\eta}(\square)
$$

The expressions on the right hand side above are built with the help of the operators $G_{j}^{\eta}(\square) Q_{j}^{*}, C^{(j), L^{J} \eta}(\square)$ and we need some of their basic properties. They are gathered in the following

Lemma 2.4. There exist positive constants $c_{0}, \delta_{0}$, and for $\alpha<1$, there exists a constant $c_{1}$, such that

$$
\begin{gathered}
\left|\left(G_{j}(\square) Q_{j}^{*}\right)(x, y)\right|,\left|\left(\partial_{\mu}^{L^{-j}} G_{j}(\square) Q_{j}^{*}\right)(x, y)\right| \leqq c_{0} e^{-\delta_{0}|x-y|}, \\
\frac{1}{\left|x-x^{\prime}\right|^{\alpha}}\left|\left(\partial_{\mu}^{L^{-j}} G_{j}(\square) Q_{j}^{*}\right)(x, y)-\left(\partial_{\mu}^{L^{-j}} G_{j}(\square) Q_{j}^{*}\right)\left(x^{\prime}, y\right)\right| \\
\leqq c_{1} e^{-\delta_{0} \operatorname{dist}\left(\left\{x, x^{\prime}\right\}, y\right)}, \\
\left|C^{(j)}\left(\square ; y, y^{\prime}\right)\right| \leqq c_{0} e^{-\delta_{0}\left|y-y^{\prime}\right|},
\end{gathered}
$$

for arbitrary non-negative integer $j$, arbitrary, rectangular parallelepiped $\square \subset L^{-j} Z^{d}$ built of large blocks, and $x, x^{\prime} \in \square, y, y^{\prime} \in \square^{(j)}=\square \cap Z^{d}$.

Using this lemma we will prove Lemma 2.2 for the propagator $G_{k}(\square)$. Let us start with the proof of the inequality (2.16). We use (2.34) with $j^{\text {th }}$ term rescaled to the $L^{-j}$-lattice:

$$
\begin{aligned}
& \frac{1}{\left|x-x^{\prime}\right|^{\alpha}}\left(\left(\partial_{\mu}^{\eta} G_{k}(\square) f\right)(x)-\left(\partial_{\mu}^{\eta} G_{k}(\square) f\right)\left(x^{\prime}\right)\right) \\
& =\frac{\eta^{1-\alpha}}{\left|\eta^{-1} x-\eta^{-1} x^{\prime}\right|^{\alpha}}\left(\left(\partial_{\mu}^{1} C^{(0)}\left(\eta^{-1} \square\right) f\right)\left(\eta^{-1} x\right)-\left(\partial_{\mu}^{1} C^{(0)}\left(\eta^{-1} \square\right) f\right)\left(\eta^{-1} x^{\prime}\right)\right) \\
& \quad+\sum_{j=1}^{k-1} a_{j}^{2} \sum_{y \in\left(L^{\prime} \eta\right)^{-1} \square^{(j)}} \frac{\left(L^{j} \eta\right)^{1-\alpha}}{\left|\left(L^{j} \eta\right)^{-1} x-\left(L^{j} \eta\right)^{-1} x^{\prime}\right|^{\alpha}} \\
& \quad \cdot\left(\left(\partial_{\mu}^{L^{-j}} G_{j}\left(\left(L^{j} \eta\right)^{-1} \square\right) Q_{j}^{*}\right)\left(\left(L^{j} \eta\right)^{-1} x, y\right)-\left(\partial_{\mu}^{L^{-j}} G_{j}\left(\left(L^{j} \eta\right)^{-1} \square\right) Q_{j}^{*}\right)\left(\left(L^{j} \eta\right)^{-1} x^{\prime}, y\right)\right) \\
& \quad \cdot\left(C^{(j)}\left(\left(L^{j} \eta\right)^{-1} \square\right) Q_{j} G_{j}\left(\left(L^{j} \eta\right)^{-1} \square\right) f\right)(y), \quad x, x^{\prime} \in \square,
\end{aligned}
$$

hence Lemma 2.4 implies

$$
\begin{aligned}
& \frac{1}{\left|x-x^{\prime}\right|^{\alpha}}\left|\left(\partial_{\mu}^{\eta} G_{k}(\square) f\right)(x)-\left(\partial_{\mu}^{\eta} G_{k}(\square) f\right)\left(x^{\prime}\right)\right| \\
& \leqq \eta^{1-\alpha} 4 c_{0} O(1)\|f\|_{\infty} \\
& \quad+\sum_{j=1}^{k-1} a_{j}^{2}\left(L^{j} \eta\right)^{1-\alpha} c_{1} \sum_{y \in\left(L^{\prime} \eta\right)^{-1} \square^{(j)}} e^{-\delta_{0} \operatorname{dist}\left(\left\{\left(L^{\prime} \eta\right)^{-1} x,\left(L^{j} \eta\right)^{-1} x^{\prime}\right\}, y\right)} \\
& \quad \cdot\left|\left(C^{(j)}\left(\left(L^{j} \eta\right)^{-1} \square\right) Q_{j} G_{j}\left(\left(L^{j} \eta\right)^{-1} \square\right) f\right)(y)\right| \\
& \leqq O(1) \sum_{j=0}^{k-1}\left(L^{j} \eta\right)^{1-\alpha}\|f\|_{\infty} \leqq c_{1}^{\prime}\|f\|_{\infty},
\end{aligned}
$$


where the constant $c_{1}^{\prime}$ is built of $c_{0}, c_{1}, \sum_{x \in Z^{d}} e^{-\delta_{0}|x|}, \sum_{j=1}^{\infty}\left(L^{-j}\right)^{1-\alpha}$. We estimate in the same way $\left|\left(G_{k}(\square) f\right)(x)\right|,\left|\left(\partial_{\mu}^{\eta} G_{k}(\square) f\right)(x)\right|$, and we get (2.16).

Remark. Let us mention here that the above method can be used also to prove pointwise estimates of $G_{k}(\square), G_{k}(\square, A)$, and even $G_{k}(\Omega, A)$, their derivatives and "Hölder-derivatives".

Now we will prove the inequality (2.17) for $G_{k}(\square)$. For $q=p=\infty$, it is a special case of (2.16), but for lack of dependence on $\alpha$, and was proved above. For $q=p=1$ we get it by duality argument, i.e. using the fact that the space $L^{\infty}(\square)$ is adjoint to $L^{1}(\square)$. The Riesz-Thorin Theorem implies it for arbitrary $q=p$ from $[1, \infty]$. (For Riesz-Thorin Theorem see Ref. [6].)

Now we will prove that the operators $G_{k}(\square), \partial_{\mu}^{\eta} G_{k}(\square), G_{k}(\square) \partial_{\mu}^{\eta *}$ are bounded operators from $L^{p_{1}}(\square)$ with $p_{1}>d$ to $L^{\infty}(\square)$. We will use again the representation (2.34). Let us consider for example the operator $G_{k}(\square) \partial_{\mu}^{\eta *}$ :

$$
\begin{aligned}
& \left(G_{k}(\square) \partial_{\mu}^{\eta *} f\right)(x)=\eta\left(C^{(0)}\left(\eta^{-1} \square\right) \partial_{\mu}^{1 * f}\right)\left(\eta^{-1} x\right) \\
& \quad+\sum_{j=1}^{k-1} a_{j}^{2} L^{j} \eta\left(G_{j}\left(\left(L^{j} \eta\right)^{-1} \square\right) Q_{j}^{*} C^{(j)}\left(\left(L^{j} \eta\right)^{-1} \square\right) Q_{j} G_{j}\left(\left(L^{j} \eta\right)^{-1} \square\right) \partial_{\mu}^{L^{-j} *} f\right) \\
& \quad \cdot\left(\left(L^{j} \eta\right)^{-1} x\right)
\end{aligned}
$$

Hence

$$
\begin{aligned}
& \left|\left(G_{k}(\square) \partial_{\mu}^{\eta *} f\right)(x)\right| \leqq \eta 2 c_{0} O(1) \eta^{-d / p_{1}}\|f\|_{p_{1}} \\
& \quad+\sum_{j=1}^{k-1} O(1) L^{j} \eta \sum_{y \in\left(L^{j} \eta\right)^{-1} \square^{(j)}} e^{-\delta_{0} \mid\left(L^{\prime} \eta\right)^{-1} x-y}\left|\left(Q_{j} G_{j}\left(\left(L^{j} \eta\right)^{-1} \square\right) \partial_{\mu}^{L^{-j} *} f\right)(y)\right| \\
& \quad \leqq O(1) \eta^{1-d / p_{1}}\|f\|_{p_{1}}+\sum_{j=1}^{k-1} O(1)\left(L^{j} \eta\right)^{1-d / p_{1}}\|f\|_{p_{1}} \leqq c_{2}^{\prime}\|f\|_{p_{1}}
\end{aligned}
$$

for $x \in \square, p_{1}>d$, where the constant $c_{2}^{\prime}$ is built of $c_{0}, \sum_{x \in Z^{d}} e^{-\delta_{0}|x|}, \sum_{j=1}^{\infty}\left(L^{-j}\right)^{1-d / p_{1}}$. Again by the duality argument we get that the operator $G_{k}(\square)$ and its first order derivatives are bounded operators from $L^{1}(\square)$ to $L^{p^{\prime}}(\square), p_{1}^{\prime-1}+p_{1}^{-1}=1$. The Riesz-Thorin Theorem gives us finally (2.17) for $G_{k}(\square)$ and for all $p, q$ described in the figure

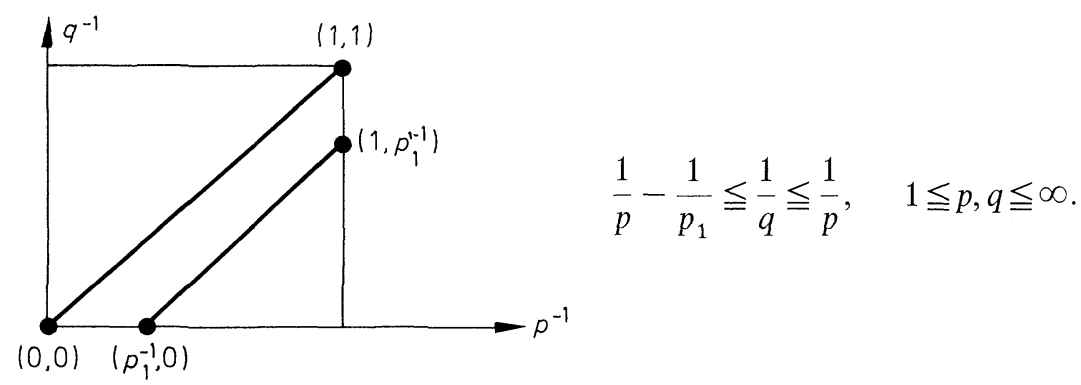

Thus Lemma 2.2 is proved, or rather reduced to Lemma 2.4. 
Proof of Lemma 2.4. At first let us notice that the inequality (2.37) concerning $C^{(j)}(\square)$ is a special case of Proposition 2.3. The proof of this proposition will be given in the next section and is based on Corollary 2.3 only. Thus we can assume that (2.37) is proved.

The proof of $(2.35),(2.36)$ can be reduced again to a simpler case. This part of the argument is valid for an arbitrary rectangular parallelepiped $\square$ built of unit blocks, so the inequalities are valid for all such sets. Let us denote $\xi=L^{-j}$. We represent $G_{j}(\square)$ with the help of the propagator $G_{j}$ with free boundary conditions on $\xi Z^{d}$ using the multiple reflection method. If $\square$ is written as $\square=\left\{x \in \xi Z^{d}: 0 \leqq x_{\mu} \leqq M_{\mu}, \mu=1, \ldots, d\right\}$, then

$$
\begin{aligned}
G_{j}\left(\square ; x, x^{\prime}\right)= & G_{j}\left(x, x^{\prime}\right)+\sum_{\mu=1}^{d} G_{j}\left(x,\left(x_{1}^{\prime}, \ldots,-x_{\mu}^{\prime}-\xi, \ldots, x_{d}^{\prime}\right)\right) \\
& +\sum_{\mu=1}^{d} G_{j}\left(x,\left(x_{1}^{\prime}, \ldots, 2 M_{\mu}-\xi-x_{\mu}^{\prime}, \ldots, x_{d}^{\prime}\right)+\ldots\right.
\end{aligned}
$$

Using this representation it is enough to prove (2.35), (2.36) for the propagator $G_{j}$.

Now we will construct an explicit representation for $G_{j}$. Let us introduce a Fourier transform on $\xi Z^{d}$ by the formulas

$$
\tilde{f}(p)=\sum_{x} \xi^{d} e^{-i p \cdot x} f(x), \quad f(x)=(2 \pi)^{-d} \int_{|p| \leqq \pi / \xi} e^{i x \cdot p} \tilde{f}(p) .
$$

We apply it to the basic equation

$$
\left(-\Delta^{\xi}+m_{j}^{2}+a_{j} Q_{j}^{*} Q_{j}\right) \phi_{0}=f .
$$

Defining the propagator $G_{j}, \phi_{0}=G_{j} f$, we get

$$
\begin{gathered}
\Delta^{\xi}(p) \tilde{\phi}_{0}(p)+a_{j} u_{j}(p) \sum_{l^{\prime}} \overline{u_{j}\left(p^{\prime}+l^{\prime}\right)} \tilde{\phi}_{0}\left(p^{\prime}+l^{\prime}\right)=\tilde{f}(p), \\
u_{j}(p)=\prod_{\mu=1}^{d} \frac{\frac{e^{-i p_{\mu}}-1}{e^{-i \xi p_{\mu}}-1}}{\xi}, \quad \Delta^{\xi}(p)=\sum_{\mu=1}^{d}\left|\frac{e^{-i \xi p_{\mu}}-1}{\xi}\right|^{2}+m_{j}^{2}, \\
p^{\prime} \in\left[-\pi, \pi\left[{ }^{d}, \quad l^{\prime}=\left(l_{1}^{\prime}, \ldots, l_{d}^{\prime}\right), \quad l_{\mu}^{\prime}=2 \pi m_{\mu}^{\prime},\right.\right. \\
m_{\mu}^{\prime} \text { is an integer, } \quad-\frac{L^{j}-1}{2} \leqq m_{\mu}^{\prime} \leqq \frac{L^{j}-1}{2} \text { for } L \text { odd }, \\
\frac{-L^{j}}{2} \leqq m_{\mu}^{\prime}<\frac{L^{j}}{2} \text { for } L \text { even. }
\end{gathered}
$$

Solving this equation we obtain the following formula :

$$
\begin{aligned}
\tilde{\phi}_{0}\left(p^{\prime}+l\right)= & \frac{1}{\Delta^{\xi}\left(p^{\prime}+l\right)} \tilde{f}\left(p^{\prime}+l\right)-\frac{u_{j}\left(p^{\prime}+l\right)}{\Delta^{\xi}\left(p^{\prime}+l\right)} \\
& \cdot \frac{a_{j}}{a_{j} \sum_{l^{\prime}} \frac{\left|u_{j}\left(p^{\prime}+l^{\prime}\right)\right|^{2}}{\Delta^{\xi}\left(p^{\prime}+l^{\prime}\right)}+1} \sum_{l^{\prime}} \frac{\overline{u_{j}\left(p^{\prime}+l^{\prime}\right)}}{\Delta^{\xi}\left(p^{\prime}+l^{\prime}\right)} \tilde{f}\left(p^{\prime}+l^{\prime}\right) .
\end{aligned}
$$


To calculate $G_{j} Q_{j}^{*}$, we take $f=Q_{j}^{*} g$ in this formula, $g$ is a function on the unit lattice, $\tilde{f}(p)=u_{j}(p) \tilde{g}\left(p^{\prime}\right), \tilde{g}\left(p^{\prime}\right)=\sum_{y} e^{-i p^{\prime} \cdot y} g(y)$, and we get

$$
\widetilde{\left(G_{j} Q_{j}^{*} g\right)}\left(p^{\prime}+l\right)=\frac{u_{j}\left(p^{\prime}+l\right)}{\Delta^{\xi}\left(p^{\prime}+l\right)} \frac{1}{a_{j} \sum_{l^{\prime}} \frac{\left|u_{j}\left(p^{\prime}+l^{\prime}\right)\right|^{2}}{\Delta^{\xi}\left(p^{\prime}+l^{\prime}\right)}+1} \tilde{g}\left(p^{\prime}\right) .
$$

From this we obtain finally the following formula for $\left(G_{i} Q_{i}^{*}\right)(x, y)$,

$$
\begin{aligned}
\left(G_{j} Q_{j}^{*}\right)(x, y)= & (2 \pi)^{-d} \int_{\left|p^{\prime}\right| \leqq \pi} d p^{\prime} \\
& \cdot \sum_{l} \frac{e^{i\left(p^{\prime}+l\right) \cdot(x-y)}}{a_{j} \sum_{l^{\prime}} \frac{\left|u_{j}\left(p^{\prime}+l^{\prime}\right)\right|^{2}}{\Delta^{\xi}\left(p^{\prime}+l^{\prime}\right)}+1} \frac{u_{j}\left(p^{\prime}+l\right)}{\Delta^{\xi}\left(p^{\prime}+l\right)} .
\end{aligned}
$$

We are now ready to prove the inequalities (2.35), (2.36); for example, let us take the expression on the left hand side of $(2.36)$ :

$$
\begin{aligned}
& \frac{1}{\left|x-x^{\prime}\right|^{\alpha}}\left(\left(\partial_{\mu}^{\xi} G_{j} Q_{j}^{*}\right)(x, y)-\left(\partial_{\mu}^{\xi} G_{j} Q_{j}^{*}\right)\left(x^{\prime}, y\right)\right) \\
& =(2 \pi)^{-d} \int_{\left|p^{\prime}\right| \leqq \pi} d p^{\prime} \\
& \\
& \quad \sum_{l} \frac{\frac{1}{\left|x-x^{\prime}\right|^{\alpha}}\left(e^{i\left(p^{\prime}+l\right) \cdot\left(x-x^{\prime}\right)}-1\right) e^{i\left(p^{\prime}+l\right)\left(x^{\prime}-y\right)}}{a_{j} \sum_{l^{\prime}} \frac{\left|u_{j}\left(p^{\prime}+l^{\prime}\right)\right|^{2}}{\Delta^{\xi}\left(p^{\prime}+l^{\prime}\right)} \Delta^{1}\left(p^{\prime}\right)+\Delta^{1}\left(p^{\prime}\right)} \\
& \quad \frac{\partial_{\mu}^{\xi}\left(p^{\prime}+l\right) u_{j}\left(p^{\prime}+l\right) \Delta^{1}\left(p^{\prime}\right)}{\Delta^{\xi}\left(p^{\prime}+l\right)},
\end{aligned}
$$

where

$$
\Delta^{1}\left(p^{\prime}\right)=\sum_{\mu=1}^{d}\left|e^{-i p_{\mu}^{\prime}}-1\right|^{2}+m_{j}^{2}, \quad \partial_{\mu}^{\xi}(p)=\frac{e^{i \xi p_{\mu}}-1}{\xi},
$$

and we have assumed that $\left|x^{\prime}-y\right| \leqq|x-y|$. It is easy to prove that the underintegral expression is bounded because

$$
\begin{aligned}
& O(1) \prod_{\mu=1}^{d} \frac{\left|p_{\mu}^{\prime}\right|}{\left|p_{\mu}^{\prime}+l_{\mu}\right|} \leqq\left|u_{j}\left(p^{\prime}+l\right)\right| \leqq O(1) \prod_{\mu=1}^{d} \frac{\left|p_{\mu}^{\prime}\right|}{\left|p_{\mu}^{\prime}+l_{\mu}\right|} \\
& \leqq O(1) \prod_{\mu=1}^{d} \frac{1}{1+\left|l_{\mu}\right|}, \\
& O(1) \frac{\left|p^{\prime}\right|^{2}+m_{j}^{2}}{\left|p^{\prime}+l\right|^{2}+m_{j}^{2}} \leqq \frac{\Delta^{1}(p)}{\Delta^{\xi}\left(p^{\prime}+l\right)} \leqq O(1) \frac{\left|p^{\prime}\right|^{2}+m_{j}^{2}}{\left|p^{\prime}+l\right|^{2}+m_{j}^{2}}, \\
&\left|\partial_{\mu}^{\xi}\left(p^{\prime}+l\right)\right| \leqq O(1)\left|p_{\mu}^{\prime}+l_{\mu}\right| \\
& \frac{1}{\left|x-x^{\prime}\right|^{\mid}}\left|e^{i\left(p^{\prime}+l\right) \cdot\left(x-x^{\prime}\right)}-1\right| \leqq O(1)\left|p^{\prime}+l\right|^{\alpha},
\end{aligned}
$$


hence

$$
\begin{aligned}
a_{j} \sum_{l^{\prime}} \frac{\left|u_{j}\left(p^{\prime}+l^{\prime}\right)\right|^{2}}{\Delta^{\xi}\left(p^{\prime}+l^{\prime}\right)} \Delta^{1}\left(p^{\prime}\right)+\Delta^{1}\left(p^{\prime}\right) & \geqq a_{j} \frac{\left|u_{j}\left(p^{\prime}\right)\right|}{\Delta^{\xi}\left(p^{\prime}\right)} \Delta^{1}\left(p^{\prime}\right) \\
& \geqq O(1)>0,
\end{aligned}
$$

and we have

|(the expression under the integral in (2.49))|

$$
\begin{aligned}
& \leqq \sum_{l} O(1)\left|p^{\prime}+l^{\alpha}\right| p_{\mu}^{\prime}+l_{\mu} \mid \frac{\left|p^{\prime}\right|^{2}+m_{j}^{2}}{\mid p^{\prime}+l^{2}+m_{j}^{2}} \prod_{\mu=1}^{d} \frac{1}{1+\left|l_{\mu}\right|} \\
& \leqq O(1)+\sum_{l \neq 0} O(1) \frac{1}{\left|p^{\prime}+l\right|^{1-\alpha}} \prod_{\mu=1}^{d} \frac{1}{1+\left|l_{\mu}\right|} \\
& \leqq O(1)+O(1)\left(\sum_{l \in Z} \frac{1}{\left(1+\left|l_{\mu}\right|\right)^{1+(1-\alpha) / d}}\right)^{d} \leqq O(1), \\
& \text { the constant depends on } \quad \alpha<1 .
\end{aligned}
$$

The expression is a function of $p^{\prime}$ and can be extended as an analytic function to some neighbourhood of $[-\pi, \pi]^{d}$. It is more troublesome, but equally elementary, to prove that this neighbourhood can be chosen independently of $j$ and that the expression is bounded also in this neighbourhood. Shifting the domain of integration in (2.49) into a complex domain in the direction of the vector $x^{\prime}-y$, we can bound the left hand side of (2.49) by a constant depending on $\alpha$ multiplied by the exponential factor $e^{-\delta_{0}\left|x^{\prime}-y\right|}=e^{-\delta_{0} \text { dist }\left(\left\{x, x^{\prime}\right\}, y\right)}$. We get the inequality (2.36). The inequalities (2.35) are proved in the same way and constants depend on $d$ only.

Thus we have proved Lemma 2.4 , hence also theTheorem.

\section{Proof of Proposition 2.3 of [1]}

The proof will be based on explicit representation for the propagators $C_{A}^{(k)}(\Omega, A)$ in terms of $G_{k}(\Omega, A)$ or related propagators. We have the following equality

$$
\begin{aligned}
& C_{\Lambda}^{(k)}\left(\Omega, A ; y, y^{\prime}\right)=\left(\left.\left(\Delta^{(k)}(\Omega, A)+a L^{-2} P(A)\right)\right|_{\Lambda}\right)^{-1}\left(y, y^{\prime}\right) \\
&=\left.\left(Z_{\Lambda}^{(k)}(\Omega, A)\right)^{-1} \int d \psi\right|_{\Lambda}\left(\frac{a L^{d-2}}{2 \pi}\right)^{\frac{N\left|\Lambda^{\prime}\right|}{2}} \\
& \quad \cdot \exp \left[-\frac{1}{2} a L^{d-2} \sum_{z \in \Lambda^{\prime}}|(Q(A) \psi)(z)|^{2}-\frac{1}{2}\left\langle\psi, \Delta^{(k)}(\Omega, A) \psi\right\rangle\right] \psi(y) \psi^{*}\left(y^{\prime}\right) \\
&=\left.\left.\left(Z_{\Lambda}^{(k)}(\Omega, A)\right)^{-1}\left(\frac{a L^{d-2}}{2 \pi}\right)^{\frac{N\left|\Lambda^{\prime}\right|}{2}} \int d \psi\right|_{\Lambda} \psi(y) \psi^{*}\left(y^{\prime}\right)\left(Z_{k}(\Omega, A)\right)^{-1} \int d \phi\right|_{\Omega}\left(\frac{a_{k}}{2 \pi}\right)^{\frac{N\left|\Omega^{(k)}\right|}{2}} \\
& \cdot \exp \left[-\frac{1}{2} a L^{d-2} \sum_{z \in \Lambda^{\prime}}|(Q(A) \psi)(z)|^{2}\right. \\
&\left.-\frac{1}{2} a_{k} \sum_{y \in \Omega^{(k)}}\left|(\Lambda \psi)(y)-\left(Q_{k}(A) \phi\right)(y)\right|^{2}-\frac{1}{2}\left\langle\phi,\left(-\Delta_{A, \Omega}^{\eta, N}+m_{k}^{2}\right) \phi\right\rangle\right] \\
&=\left(Z_{\Lambda}^{(k)}(\Omega, A) Z_{k}(\Omega, A)\right)^{-1}\left(\frac{a L^{d-2}}{2 \pi}\right)^{\frac{N\left|\Lambda^{\prime}\right|}{2}}\left(\frac{a_{k}}{2 \pi}\right)^{\frac{N \mid \Omega^{(k) \mid}}{2}}
\end{aligned}
$$




$$
\begin{aligned}
& \left.\cdot \int d \phi\right|_{\Omega}\left\{\left.\int d \psi\right|_{\Lambda}\left(\psi(y)+\left(Q_{k}(A) \phi\right)(y)\right)\left(\psi^{*}\left(y^{\prime}\right)+\left(Q_{k}(A) \phi\right)^{*}\left(y^{\prime}\right)\right)\right. \\
& \cdot \exp \left[-\frac{1}{2} a_{k} \sum_{y \in \Lambda}|\psi(y)|^{2}-\frac{1}{2} a L^{d-2} \sum_{z \in \Lambda^{\prime}}|(Q(A) \psi)(z)|^{2}\right. \\
& \left.\left.-a L^{d-2} \sum_{z \in \Lambda^{\prime}}(Q(A) \psi)(z)\left(Q_{k+1}(A) \phi\right)(z)\right]\right\} \\
& \cdot \exp \left[-\frac{1}{2} a L^{d-2} \sum_{z \in \Lambda}\left|\left(Q_{k+1}(A) \phi\right)(z)\right|^{2}\right. \\
& \left.-\frac{1}{2} a_{k} \sum_{y \in \Omega^{(k)} \backslash \Lambda}\left|\left(Q_{k}(A) \phi\right)(y)\right|^{2}-\frac{1}{2}\left\langle\phi,\left(-\Delta_{A, \Omega}^{\eta, N}+m_{k}^{2}\right) \phi\right\rangle\right] .
\end{aligned}
$$

The integral in the curly bracket $\{\ldots\}$ factorizes into integrals with respect to $\left.\psi\right|_{B(z)}$. In these we make a gauge transformation $\psi(y) \rightarrow U\left(A\left(\Gamma_{y, z}\right)\right) \psi(y)$, and we get integrals which can be factorized with respect to components of $\psi$. After this we have to compute integrals of the form

$\left.\int d \psi\right|_{B(z)}\left\{1, \psi(y), \psi(y) \psi\left(y^{\prime}\right)\right\}$

$$
\cdot \exp \left[-\frac{1}{2} a_{k} \sum_{y \in B(z)} \psi^{2}(y)-\frac{1}{2} a L^{d-2}\left(L^{-d} \sum_{y \in B(z)} \psi(z)\right)^{2}-a L^{d-2} L^{-d} \sum_{y \in B(z)} \psi(y) C\right],
$$

where $C$ is a component of $\left(Q_{k+1}(A) \phi\right)(z)$.

We apply the general formula

$$
\begin{aligned}
\int \prod_{j=1}^{N} d x_{j}\left\{1, x_{j}, x_{j} x_{k}\right\} \exp \left[-\frac{1}{2} A\left(\sum_{j=1}^{N} x_{j}\right)^{2}-\frac{1}{2} B \sum_{j=1}^{N} x_{j}^{2}+C \sum_{j=1}^{N} x_{j}\right] \\
=\left\{1, \frac{C}{N A+B},\left(\frac{C}{N A+B}\right)^{2}-\frac{A}{B(N A+B)}+\frac{\delta_{j, k}}{B}\right\} \\
\cdot(2 \pi)^{N / 2}\left((N A+B) B^{N-1}\right)^{-1 / 2} \exp \left(\frac{1}{2} \frac{N C^{2}}{N A+B}\right),
\end{aligned}
$$

and using the recursive relation $a_{k+1}=\frac{a a_{k}}{a L^{-2}+a_{k}}$, we get

$$
\begin{aligned}
C_{\Lambda}^{(k)}\left(\Omega, A ; y, y^{\prime}\right)= & \left.Z^{-1} \int d \phi\right|_{\Omega}\left\{\left[-\frac{a_{k+1}}{a_{k}} L^{-2}\left(Q^{*}(A) Q_{k+1}(A) \phi\right)(y)+\left(Q_{k}(A) \phi\right)(y)\right]\right. \\
& \cdot\left[-\frac{a_{k+1}}{a_{k}} L^{-2}\left(Q^{*}(A) Q_{k+1}(A) \phi\right)^{*}\left(y^{\prime}\right)+\left(Q_{k}(A) \phi\right)^{*}\left(y^{\prime}\right)\right] \\
& \left.-\frac{a_{k+1}}{a_{k}^{2}} L^{-2} P\left(A ; y, y^{\prime}\right)+\frac{\delta_{y, y^{\prime}}}{a_{k}}\right\} \\
& \cdot \exp \left[-\frac{1}{2} a_{k} \sum_{y \in \Omega^{(k)} \backslash \Lambda}\left|\left(Q_{k}(A) \phi\right)(y)\right|^{2}\right. \\
& \left.-\frac{1}{2} a_{k+1} L^{d-2} \sum_{z \in \Lambda^{\prime}}\left|\left(Q_{k+1}(A) \phi\right)(z)\right|^{2}-\frac{1}{2}\left\langle\phi,\left(-\Delta_{A, \Omega}^{\eta, N}+m_{k}^{2}\right) \phi\right\rangle\right],
\end{aligned}
$$

$Z$ is of course a normalization factor. Denoting

$$
G_{k}(\Omega, \Lambda, A)=\left(-\Delta_{A, \Omega}^{\eta, N}+m_{k}^{2}+a_{k} P_{k}(A)\left(\Omega \backslash B^{k}(\Lambda)\right)+a_{k+1} L^{-2} P_{k+1}(A) B^{k}(\Lambda)\right)^{-1},
$$


we have finally

$$
\begin{aligned}
C_{\Lambda}^{(k)}\left(\Omega, A ; y, y^{\prime}\right)= & \left(\left[-\frac{a_{k+1}}{a_{k}} L^{-2} Q^{*}(A) Q_{k+1}(A)+Q_{k}(A)\right] G_{k}(\Omega, \Lambda, A)\right. \\
& \left.\cdot\left[-\frac{a_{k+1}}{a_{k}} L^{-2} Q_{k+1}^{*}(A) Q(A)+Q_{k}^{*}(A)\right]\right)\left(y, y^{\prime}\right) \\
& -\frac{a_{k+1}}{a_{k}^{2}} L^{-2} P\left(A ; y, y^{\prime}\right)+\frac{\delta_{y, y^{\prime}}}{a_{k}} .
\end{aligned}
$$

A composition of an operator $T$ defined on the $\eta$-lattice with $Q_{k}^{*}(A)$ or $Q_{k+1}^{*}(A) Q(A)$ can be interpreted as the action of the operator $T$ on properly defined functions on the $\eta$-lattice. More exactly if we define the functions $q_{k}(y)$, $q_{k+1}(y)$ by the formulas

$$
\begin{aligned}
q_{k}(y, x)= & \text { a column of } U\left(A\left(\Gamma_{x, y}^{(k)}\right)\right) \text { for } x \in B^{k}(y), 0 \text { otherwise, } \\
q_{k+1}(y, x)= & \text { a column of } L^{-d} U\left(A\left(\Gamma_{x, z}^{(k+1)} \cup \Gamma_{z, y}\right)\right) \text { for } x \in B^{k+1}(z), \\
& z \text { such that } y \in B(z), 0 \text { otherwise, }
\end{aligned}
$$

then we have

$$
\begin{aligned}
C_{\Lambda}^{(k)}\left(\Omega, A ; y, y^{\prime}\right) \\
=\left\langle-\frac{a_{k+1}}{a_{k}} L^{-2} q_{k+1}(y)+q_{k}(y), G_{k}(\Omega, \Lambda, A)\left(-\frac{a_{k+1}}{a_{k}} L^{-2} q_{k+1}\left(y^{\prime}\right)+q_{k}\left(y^{\prime}\right)\right)\right\rangle \\
\quad-\frac{a_{k+1}}{a_{k}^{2}} L^{-2} P\left(A ; y, y^{\prime}\right)+\frac{\delta_{y, y^{\prime}}}{a_{k}} .
\end{aligned}
$$

The $L^{2}$-norms of the functions $q_{k}(y), q_{k+1}(y)$ are equal to $1, L^{-d / 2}$ and their supports are in $B^{k}(y), B^{k+1}(z(y))$ respectively, so the inequalities (1.11), (1.12), and (1.16) are simple consequences of Corollary 2.3. At this moment let us mention that the corollary was proved for the propagator $G_{k}(\Omega, A)$, not for $G_{k}(\Omega, A, A)$, but the only difference between these operators is that $G_{k}(\Omega, A, A)$ is, according to (3.5), defined by $L$-blocks on unit scale $\Lambda$, i.e. blocks $B^{k+1}(z), z \in \Lambda^{\prime}$, instead of unit blocks $B^{k}(y), y \in \Lambda$, for $G_{k}(\Omega, A)$. This difference is unessential and the whole proof of Corollary 2.3 goes on with only slight changes, the most important is that in the inequality (2.27) for $\Delta=B^{k+1}(z), z \in \Lambda^{\prime}$, we have to replace $\min \left\{\pi^{2}, a_{k}\right\}$ by $\min \left\{\pi^{2} L^{-2}, a_{k+1} L^{-2}\right\}$, but this is also some positive constant only depending additionally on $L$. Generally we have to expect that bounds on $C_{A}^{(k)}(\Omega, A)$ will depend on $L$.

Now to prove the inequality (1.14) we have to estimate the difference $G_{k}(\Omega, \Lambda, A)-G_{k+1}^{\eta}(\Omega, A)$. This is easy because on the basis of (3.5) we have

$$
\begin{aligned}
& G_{k}(\Omega, \Lambda, A)-G_{k+1}^{\eta}(\Omega, A) \\
& \quad=G_{k}(\Omega, \Lambda, A)\left[a_{k} P_{k}(A)-a_{k+1} L^{-2} P_{k+1}(A)\right]\left(\Omega \backslash B^{k}(\Lambda)\right) G_{k+1}^{\eta}(\Omega, A),
\end{aligned}
$$


and for $f, f^{\prime} \in L^{2}\left(B^{k}(\Lambda)\right)$, we get

$$
\begin{aligned}
& \left|\left\langle f,\left(G_{k}(\Omega, \Lambda, A)-G_{k+1}^{\eta}(\Omega, A)\right) f^{\prime}\right\rangle\right| \leqq c_{0}\|f\|_{2}\left\|f^{\prime}\right\|_{2} \\
& \quad \cdot \exp \left[-\delta_{0}\left(\operatorname{dist}\left(\operatorname{supp} f, \operatorname{supp} f^{\prime}\right)+\operatorname{dist}\left(\operatorname{supp} f, B^{k}\left(\Lambda^{c}\right)\right)+\operatorname{dist}\left(\operatorname{supp} f^{\prime}, B^{k}\left(\Lambda^{c}\right)\right)\right)\right] .
\end{aligned}
$$

The function $\delta C_{A}^{(k)}(\Omega, A)$ is given by (3.8) with the operator (3.9) instead of $G_{k}(\Omega, \Lambda, A)$ and the inequality (3.10) implies (1.14). This ends the proof of Proposition I.2.3. Another proof will be given in the last section.

\section{Proof of the Lower Bound for the Quadratic Form $\Delta^{(k)}$ (Proposition 3.1')}

We will estimate the quadratic form on the left hand side of (1.18) in several ways, finally getting all the terms on the right hand side.

At first we have $G_{k}(\Omega, A) \leqq\left(\left.\left(a_{k} P_{k}(A)+m^{2}\right)\right|_{\Omega}\right)^{-1}$, and the left side of (1.18) can be estimated from below by

$$
\sum_{x \in \Omega^{(k)}}\left[a_{k}|\phi(x)|^{2}-a_{k}^{2} \phi(x) \cdot\left(Q_{k}(A)\left(\left.\left(a_{k} P_{k}(A)+m^{2}\right)\right|_{B^{k}(x)}\right)^{-1} Q_{k}^{*}(A)\right)(x, x) \phi(x)\right] .
$$

Each term of the above sum is determined by the integral

$$
\left.\int d \phi^{\prime}\right|_{B^{k}(x)} \exp \left[-\frac{1}{2} a_{k}\left|\phi(x)-\left(Q_{k}(A) \phi^{\prime}\right)(x)\right|^{2}-\frac{1}{2} m^{2} \sum_{x^{\prime} \in B^{k}(x)} \eta^{d}\left|\phi^{\prime}(x)\right|^{2}\right] .
$$

This means that the integral is equal to const $\exp \left[-\frac{1}{2}\right.$ (the term of the sum (4.1) corresponding to the point $x)$ ]. In this integral we make the gauge transformation $\phi^{\prime}\left(x^{\prime}\right)=U\left(A\left(\Gamma_{x^{\prime}, x}^{(k)}\right)\right) \phi^{\prime \prime}\left(x^{\prime}\right)$, and we integrate over the subspace orthogonal to the constant functions on $B^{k}(x)$. Then we get

$$
\begin{gathered}
\text { const } \int d \phi^{\prime \prime} \exp \left(-\frac{1}{2} a_{k}\left|\phi(x)-\phi^{\prime \prime}\right|^{2}-\frac{1}{2} m^{2}\left|\phi^{\prime \prime}\right|^{2}\right) \\
=\text { const }^{\prime} \exp \left(-\frac{1}{2}\left(a_{k}-\frac{a_{k}^{2}}{a_{k}+m^{2}}\right)|\phi(x)|^{2}\right),
\end{gathered}
$$

and hence

(the left hand side of (1.18)) $\geqq \sum_{x \in \Omega^{(k)}} \frac{a_{k} m^{2}}{a_{k}+m^{2}}|\phi(x)|^{2} \geqq \frac{a_{k}}{a_{k}+O(1)} \sum_{x \in \Omega^{(k)}} m^{2}|\phi(x)|^{2}$,

if $m^{2} \leqq O(1)$. Thus a part of the inequality (1.18) is proved.

Now let us consider the set of all positively oriented bonds $\left\langle x, x^{\prime}\right\rangle \subset \Omega^{(k)}$. This set can be represented as a sum of $2 d$ subsets $B_{i}$ with the property that each point of $\Omega^{(k)}$ belongs to at most one bond in a given subset $B_{i}$. For example we can define $B_{2 \mu-1}$ as a set of bonds $\left\langle x, x+e_{\mu}\right\rangle \subset \Omega^{(k)}$ for which the coordinate $x_{\mu}$ of the point $x$ is an odd number; similarly we define $B_{2 \mu}$ with the difference that $x_{\mu}$ is an even number. For a given $B_{i}$ there may be points which do not belong to any bond of this set. Let us denote $\Delta\left(x, x^{\prime}\right)=B^{k}(x) \cup B^{k}\left(x^{\prime}\right)$. Again separating subsets of $\Omega$ by the Neumann boundary conditions we have 
(the left hand side of (1.18))

$$
\begin{aligned}
& \geqq \sum_{\left\langle x, x^{\prime}\right\rangle \in B_{i}}\left[a_{k}|\phi(x)|^{2}+a_{k}\left|\phi\left(x^{\prime}\right)\right|^{2}-a_{k}^{2}\left\langle\phi, Q_{k}(A) G_{k}\left(\Delta\left(x, x^{\prime}\right), A\right) Q_{k}^{*}(A) \phi\right\rangle\right] \\
& \quad+\sum_{x \in \Omega^{(k)}, x \text { does not belong to a bond in } B_{l}}\left[a_{k}|\phi(x)|^{2}-a_{k}^{2} \phi(x)\right. \\
& \left.\quad \cdot\left(Q_{k}(A) G_{k}\left(B^{k}(x), A\right) Q_{k}^{*}(A)\right)(x, x) \phi(x)\right] .
\end{aligned}
$$

Each term in the second sum of the right side (4.5) is non-negative, as it follows from the proof of (4.4), so we can omit these terms. Summing the inequalities (4.5) over $B_{i}, i=1, \ldots, 2 d$, we get the inequality

(the left hand side of (1.18))

$$
\geqq \frac{1}{2 d} \sum_{\left\langle x, x^{\prime}\right\rangle \subset \Omega^{(k)}}\left[a_{k}|\phi(x)|^{2}+a_{k}\left|\phi\left(x^{\prime}\right)\right|^{2}-a_{k}^{2}\left\langle\phi, Q_{k}(A) G_{k}\left(\Delta\left(x, x^{\prime}\right), A\right) Q_{k}^{*}(A) \phi\right\rangle\right] .
$$

Now it is easily seen that to prove (1.18) it is sufficient to prove

$$
\begin{aligned}
& a_{k}|\phi(x)|^{2}+a_{k}\left|\phi\left(x^{\prime}\right)\right|^{2}-a_{k}^{2}\left\langle\phi, Q_{k}(A) G_{k}\left(\Delta\left(x, x^{\prime}\right), A\right) Q_{k}^{*}(A) \phi\right\rangle \\
& \quad \geqq \gamma_{0}^{\prime}\left|U\left(A\left(\left\langle x, x^{\prime}\right\rangle\right)\right) \phi\left(x^{\prime}\right)-\phi(x)\right|^{2}-O(1) e^{2} p^{2}(e)\left(|\phi(x)|^{2}+\left|\phi\left(x^{\prime}\right)\right|^{2}\right),
\end{aligned}
$$

with $\gamma_{0}^{\prime}$ independent of $k,\left\langle x, x^{\prime}\right\rangle$, and $A$. Then $\gamma_{0}=\frac{1}{2} \min \left\{\frac{1}{2 d} \gamma_{0}^{\prime}, \frac{a_{k}}{a_{k}+O(1)}\right\}$. We will transform the left hand side of (4.7). Using the regularity condition for $A$, we write $A=A_{0}+A^{\prime}$ on $\Delta\left(x, x^{\prime}\right)$ with $A_{0}$ constant and $A^{\prime}$ satisfying the bounds $\left|A^{\prime}\right|,\left|\partial_{\mu}^{\eta} A^{\prime}\right|$ $\leqq O(1) p(e)$. We expand the expression on the left hand side of (4.7) with respect to $A^{\prime}$ using (I.3.15), (I.3.44), and we separate terms of first order in $e$. Using Lemma 2.1 the remaining terms can be easily estimated by $O\left(e^{2} p^{2}(e)\right)\left(|\phi(x)|^{2}+\left|\phi\left(x^{\prime}\right)\right|^{2}\right)$. Now let us consider the terms of first order. Formally we could rely on the estimates of perturbative expansions in the third paper [3], but there they were derived in a different setting, the interaction terms considered on a domain far from the boundary of basic domains for propagators, so let us repeat this part of the argument. Denoting $\phi^{(k)}=a_{k} G_{k}\left(\Delta\left(x, x^{\prime}\right), A_{0}\right) Q_{k}^{*}\left(A_{0}\right) \phi$, we have the following expression

$$
\begin{aligned}
& 2 \sum_{b \subset \Delta\left(x, x^{\prime}\right)}\left[\left(D_{A_{0}}^{\eta} \phi^{(k)}\right)(b) \cdot q \phi^{(k)}\left(b_{-}\right)\right] e A_{b}^{\prime} \\
& \quad+2 a_{k} \sum_{y=x, x^{\prime}}\left(Q_{k}\left(A_{0}\right) \phi^{(k)}\right)(y) \cdot \sum_{z \in B^{k}(y)} \eta^{d} e \eta A^{\prime}\left(\Gamma_{y, z}^{(k)}\right) q U\left(A_{0}\left(\Gamma_{y, z}^{(k)}\right)\right) \phi^{(k)}(z) \\
& \quad-2 a_{k} \sum_{y=x, x^{\prime}} \phi(y) \cdot \sum_{z \in B^{k}(y)} \eta^{d} e \eta A^{\prime}\left(\Gamma_{y, z}^{(k)}\right) q U\left(A_{0}\left(\Gamma_{y, z}^{(k)}\right)\right) \phi^{(k)}(z) .
\end{aligned}
$$

Now we have to inspect closely the proof of the Lemma II.2.4. At first let us notice that we can "gauge away" the configuration $A_{0}$ using the same gauge transformation, and we get the expression (4.8) with $A_{0}=0$ and $\phi(x), \phi\left(x^{\prime}\right)$ replaced by $\phi(x), U\left(A_{0}\left(\left\langle x, x^{\prime}\right\rangle\right)\right) \phi\left(x^{\prime}\right)$ respectively. Then using II.2.75 we have

$$
\begin{aligned}
\phi^{(k)}(z) & =\frac{1}{1+m^{2}} \phi(x)+O\left(U\left(A_{0}\left(\left\langle x, x^{\prime}\right\rangle\right)\right) \phi\left(x^{\prime}\right)-\phi(x)\right), \quad z \in \Delta\left(x, x^{\prime}\right), \\
\left(\partial^{\eta} \phi^{(k)}\right)(b) & =O\left(U\left(A_{0}\left(\left\langle x, x^{\prime}\right\rangle\right)\right) \phi\left(x^{\prime}\right)-\phi(x)\right), \quad b \in \Delta\left(x, x^{\prime}\right),
\end{aligned}
$$


and this implies the following estimate

$\mid($ the expression (4.8))|

$$
\begin{aligned}
\leqq & \mid \frac{4 a_{k}}{\left(1+m^{2}\right)^{2}}[\phi(x) \cdot q \phi(x)] \sum_{y=x, x^{\prime}} \sum_{z \in B^{k}(y)} \eta^{d} e \eta A^{\prime}\left(\Gamma_{y, z}\right) \\
& -\frac{4 a_{k}}{1+m^{2}}[\phi(x) \cdot q \phi(x)] \sum_{y=x, x^{\prime}} \sum_{z \in B^{k}(y)} \eta^{d} e \eta A^{\prime}\left(\Gamma_{y, z}\right) \mid \\
& +O(1) e p(e)\left|U\left(A_{0}\left(\left\langle x, x^{\prime}\right\rangle\right)\right) \phi\left(x^{\prime}\right)-\phi(x)\right|\left(|\phi(x)|+\left|\phi\left(x^{\prime}\right)\right|\right) \\
\leqq & \delta\left|U\left(A_{0}\left(\left\langle x, x^{\prime}\right\rangle\right)\right) \phi\left(x^{\prime}\right)-\phi(x)\right|^{2}+O\left(\delta^{-1}\right) e^{2} p^{2}(e)\left(|\phi(x)|^{2}+\left|\phi\left(x^{\prime}\right)\right|^{2}\right), \\
& (\phi(x) \cdot q \phi(x)=0 \text { because } q \text { is an antisymmetric matrix }), \\
& \text { for arbitrary } \delta>0 .
\end{aligned}
$$

Thus we get

(the left hand side of (4.7))

$$
\begin{aligned}
& \geqq\left(\text { the left hand side of (4.7) with } A \text { replaced by } A_{0}\right) \\
& \quad-\delta\left|U\left(A_{0}\left(\left\langle x, x^{\prime}\right\rangle\right)\right) \phi\left(x^{\prime}\right)-\phi(x)\right|^{2}-O\left(\delta^{-1}\right) e^{2} p^{2}(e)\left(|\phi(x)|^{2}+\left|\phi\left(x^{\prime}\right)\right|^{2}\right) .
\end{aligned}
$$

Now it is sufficient to prove the inequality

(the left hand side of (4.7) with $A$ replaced by $\left.A_{0}\right) \geqq \gamma_{0}^{\prime \prime}\left|U\left(A_{0}\left(\left\langle x, x^{\prime}\right\rangle\right)\right) \phi\left(x^{\prime}\right)-\phi(x)\right|^{2}$,

because this together with (4.11) with $\delta=\frac{1}{2} \gamma_{0}^{\prime \prime}$ and

$$
\left|U\left(A_{0}\left(\left\langle x, x^{\prime}\right\rangle\right)\right) \phi\left(x^{\prime}\right)-\phi(x)\right|^{2} \geqq \frac{1}{2}\left|U\left(A\left(\left\langle x, x^{\prime}\right\rangle\right)\right) \phi\left(x^{\prime}\right)-\phi(x)\right|^{2}-O(1) e^{2} p^{2}(e)|\phi(x)|^{2},
$$

give (4.7) with $\gamma_{0}^{\prime}=\frac{1}{4} \gamma_{0}^{\prime \prime}$. Let us notice that the second term on the right hand side of (4.7) does not appear if $A=0$.

Now we will prove (4.12). At first we estimate the left side of it from below by the same expression with $m^{2}=0$. Further we "gauge out" the constant field $A$ from the expression with the simultaneous gauge transformations of $\phi: \phi(x)=\phi^{\prime \prime}(x)$, $\phi\left(x^{\prime}\right)=U\left(A\left(\left\langle x^{\prime}, x\right\rangle\right)\right) \phi^{\prime \prime}\left(x^{\prime}\right)$, as in the proof of Lemma II.2.4. Then the inequality (4.12) is transformed into the inequality

$$
a_{k}\left|\phi^{\prime \prime}(x)\right|^{2}+a_{k}\left|\phi^{\prime \prime}\left(x^{\prime}\right)\right|^{2}-a_{k}^{2}\left\langle\phi^{\prime \prime}, Q_{k} G_{k}\left(\Delta\left(x, x^{\prime}\right), 0\right) Q_{k}^{*} \phi^{\prime \prime}\right\rangle \geqq \gamma_{0}^{\prime \prime}\left|\phi^{\prime \prime}\left(x^{\prime}\right)-\phi^{\prime \prime}(x)\right|^{2} .
$$

We use again the fact that const. $\exp \left[-\frac{1}{2}(\right.$ the left side of $\left.(4.14))\right]$ is equal to the integral

$$
\begin{aligned}
& \left.\int d \phi^{\prime}\right|_{\Delta\left(x, x^{\prime}\right)} \\
& \cdot \exp \left[-\frac{1}{2} a_{k}\left|\phi^{\prime \prime}(x)-\left(Q_{k} \phi^{\prime}\right)(x)\right|^{2}-\frac{1}{2} a_{k}\left|\phi^{\prime \prime}\left(x^{\prime}\right)-\left(Q_{k} \phi^{\prime}\right)\left(x^{\prime}\right)\right|^{2}-\frac{1}{2}\left\langle\phi^{\prime},\left(-\Delta_{\Delta\left(x, x^{\prime}\right)}^{\eta, N}\right) \phi^{\prime}\right\rangle\right] .
\end{aligned}
$$

Denoting $\Delta=B^{k}(x), \Delta^{\prime}=B^{k}\left(x^{\prime}\right), \psi=\phi_{k}^{\prime \prime}\left(x^{\prime}\right)-\phi_{k}^{\prime \prime}(x)$, and making the translations $\phi^{\prime}(y)=\phi(y)+\phi^{\prime \prime}(x)$ for $y \in \Delta, \phi^{\prime}(y)=\phi(y)+\phi^{\prime \prime}(x)$ for $y \in \Delta^{\prime}$ in the above integral, we get 


$$
\begin{aligned}
& \left.\int d \phi\right|_{\Delta\left(x, x^{\prime}\right)} \\
& \quad \cdot \exp \left[-\frac{1}{2} a_{k}\left|\left(Q_{k} \phi\right)(x)\right|^{2}-\frac{1}{2}\left\langle\phi,\left(-\Delta_{\Delta}^{\eta, N}\right) \phi\right\rangle-\frac{1}{2} a_{k}\left|\left(Q_{k} \phi\right)\left(x^{\prime}\right)\right|^{2}-\frac{1}{2}\left\langle\phi,\left(-\Delta_{\Delta}^{\eta, N}\right) \phi\right\rangle\right. \\
& \left.\quad-\frac{1}{2} \sum_{b \in B\left(\left\langle x, x^{\prime}\right\rangle\right)} \eta^{d}\left|\left(\partial^{\eta} \phi\right)(b)+\eta^{-1} \psi\right|^{2}\right]=\text { const } \exp \left(-\frac{1}{2} A_{0}|\psi|^{2}\right),
\end{aligned}
$$

where $B\left(\left\langle x, x^{\prime}\right\rangle\right)$ denotes the set of all bonds of the $\eta$-lattice connecting the block $B^{k}(x)$ with the block $B^{k}\left(x^{\prime}\right)$, i.e. $B\left(\left\langle x, x^{\prime}\right\rangle\right)=\left\{b \subset T_{\eta}: b_{-} \in B^{k}(x), b_{+} \in B^{k}\left(x^{\prime}\right)\right\}$. Now we have to show that $A_{0}>0$, more exactly $A_{0} \geqq \gamma_{0}^{\prime \prime}, \gamma_{0}^{\prime \prime}>0$, and $\gamma_{0}^{\prime \prime}$ depends on $d$ and $a$ only. It is obvious that the integral on the left hand side and the function on the right hand side of (4.16) factorizes into the products of the corresponding expressions for the components of $\phi$ and $\psi$. We can assume that $\phi$ and $\psi$ are real quantities. We will use the following simple formula for the Fourier transform of the Gaussian function:

$$
\int d \psi \exp (-i \xi \psi) \exp \left(-\frac{1}{2} A_{0} \psi^{2}\right)=\sqrt{2 \pi A_{0}^{-1}} \exp \left(-\frac{1}{2} A_{0}^{-1} \xi^{2}\right) .
$$

Now it is sufficient to prove that $0<A_{0}^{-1} \leqq \gamma_{0}^{\prime \prime-1}$, and $\gamma_{0}^{\prime \prime-1}$ depends on $d$ and $a$ only. Let us calculate the Fourier transform of the integral on the left hand side of (4.16). At first we transform the last expression in the exponent:

$$
\begin{aligned}
& \sum_{b \in B\left(\left\langle x, x^{\prime}\right\rangle\right)} \eta^{d}\left|\left(\partial^{\eta} \phi\right)(b)+\eta^{-1} \psi\right|^{2}=\sum_{b \in B\left(\left\langle x, x^{\prime}\right\rangle\right)} \eta^{d} \mid\left(\partial^{\eta} \phi\right)(b) \\
& \quad-\left.\sum_{b^{\prime} \in B\left(\left\langle x, x^{\prime}\right\rangle\right)} \eta^{d-1}\left(\partial^{\eta} \phi\right)\left(b^{\prime}\right)\right|^{2}+\eta^{-1}\left|\psi+\sum_{b \in B\left(\left\langle x, x^{\prime}\right\rangle\right)} \eta^{d}\left(\partial^{\eta} \phi\right)(b)\right|^{2} .
\end{aligned}
$$

Let us introduce the notation

$$
\begin{aligned}
\langle\phi, A \phi\rangle= & a_{k}\left|\left(Q_{k} \phi\right)(x)\right|^{2}+\left\langle\phi,\left(-\Delta_{\Delta}^{\eta, N}\right) \phi\right\rangle+a_{k}\left|\left(Q_{k} \phi\right)\left(x^{\prime}\right)\right|^{2}+\left\langle\phi,\left(-\Delta_{\Delta^{\prime}}^{\eta, N}\right) \phi\right\rangle \\
& +\sum_{b \in B\left(\left\langle x, x^{\prime}\right\rangle\right)} \eta^{d}\left|\left(\partial^{\eta} \phi\right)(b)-\sum_{b^{\prime} \in B\left(\left\langle x, x^{\prime}\right\rangle\right)} \eta^{d-1}\left(\partial^{\eta} \phi\right)\left(b^{\prime}\right)\right|^{2} .
\end{aligned}
$$

The Fourier transform with respect to $\psi$ of the expression on the left hand side of (4.16) can be written in the form

$$
\begin{aligned}
\int d \psi & \exp (-i \xi \psi) \int d \phi \exp \left[-\frac{1}{2} \eta^{-1}\left|\psi+\sum_{b \in B\left(\left\langle x, x^{\prime}\right\rangle\right)} \eta^{d-1}\left(\phi\left(b_{+}\right)-\phi\left(b_{-}\right)\right)\right|^{2}-\frac{1}{2}\langle\phi, A \phi\rangle\right] \\
= & \int d \psi \exp (-i \xi \psi) \exp \left(-\frac{1}{2} \eta^{-1} \psi^{2}\right) \int d \phi \exp i \xi \sum_{b \in B\left(\left\langle x, x^{\prime}\right\rangle\right)} \eta^{d-1}\left(\phi\left(b_{+}\right)-\phi\left(b_{-}\right)\right) \\
& \cdot \exp \left(-\frac{1}{2}\langle\phi, A \phi\rangle\right) \\
= & \sqrt{2 \pi \eta} \exp \left(-\frac{1}{2} \eta \xi^{2}\right)(2 \pi)^{L^{k d}}(\operatorname{det} A)^{-1 / 2} \exp \left(-\frac{1}{2}\left\langle f_{\left\langle x, x^{\prime}\right\rangle}, A^{-1} f_{\left\langle x, x^{\prime}\right\rangle}\right\rangle \xi^{2}\right),
\end{aligned}
$$

and we get the formula for $A_{0}^{-1}$ :

$$
A_{0}^{-1}=\eta+\left\langle f_{\left\langle x, x^{\prime}\right\rangle}, A^{-1} f_{\left\langle x, x^{\prime}\right\rangle}\right\rangle
$$

where

$$
f_{\left\langle x, x^{\prime}\right\rangle}(y)=\sum_{b \in B\left(\left\langle x, x^{\prime}\right\rangle\right)} \eta^{d-1}\left(\delta^{\eta}\left(y-b_{+}\right)-\delta^{\eta}\left(y-b_{-}\right)\right) .
$$

Of course both terms on the right side of (4.21) are $\geqq 0$, and $\eta=L^{-k}, 0<\eta<1$. We 
have to estimate the second term. From (4.19) and the definition of $f_{\left\langle x, x^{\prime}\right\rangle}$ it follows that

$$
\begin{aligned}
\left\langle f_{\left\langle x, x^{\prime}\right\rangle}, A^{-1} f_{\left\langle x, x^{\prime}\right\rangle}\right\rangle & \leqq\left\langle f_{\left\langle x, x^{\prime}\right\rangle}, G_{k}(\Delta) f_{\left\langle x, x^{\prime}\right\rangle}\right\rangle+\left\langle f_{\left\langle x, x^{\prime}\right\rangle}, G_{k}\left(\Delta^{\prime}\right) f_{\left\langle x, x^{\prime}\right\rangle}\right\rangle \\
& =2 \sum_{y, y^{\prime} \in \Delta_{1}} \eta^{2(d-1)} G_{k}\left(\Delta ; y, y^{\prime}\right),
\end{aligned}
$$

where $\Delta_{1}$ is any face of the block $\Delta$, e.g. $\Delta_{1}=\left\{y \in \Delta: y_{1}=x_{1}+1-\eta\right\}$. Of course the expression on the right side of (4.22) is a constant $\gamma_{0}^{\prime \prime-1}$ depending on $d$ and $a$ only. Thus the inequality (4.14) is proved and the proof of Proposition 3.1' is completed.

Remark. The argument applied in the proof of (4.14) is an example of an application of "duality transformations". In our case this is an ordinary Fourier transform.

\section{A General Theorem on Unit Lattice Operators}

There is another way of proving Proposition I.2.3, relying on a general theorem concerning inverses to some unit lattice operators. This theorem is interesting enough in itself to devote a separate section to it.

At first let us prove that the bounds (1.11) are consequences of Proposition II.3.1'. The upper bound is quite elementary because

$$
\begin{aligned}
\Delta^{(k)}(\Omega, A)+a L^{-2} P(A) & =a_{k} I-a_{k}^{2} Q_{k}(A) G_{k}(\Omega, A) Q_{k}^{*}(A)+a L^{-2} P(A) \\
& \leqq a_{k} I+a L^{-2} Q^{*}(A) Q(A) \leqq\left(a_{k}+a L^{-2}\right) I=a \frac{a_{k}}{a_{k+1}} I .
\end{aligned}
$$

The operators are considered as defined on $L^{2}\left(\Omega^{(k)}\right)$. To prove the lower bound we apply Proposition II.3.1' to $\Delta^{(k)}(\Omega, A)$ :

$$
\begin{aligned}
& \left\langle\phi,\left(\Delta^{(k)}(\Omega, A)+a L^{-2} P(A)\right) \phi\right\rangle \geqq \gamma_{0} \sum_{\left\langle x, x^{\prime}\right\rangle \subset \Omega^{(k)}}\left|U\left(A\left(\left\langle x, x^{\prime}\right\rangle\right)\right) \phi\left(x^{\prime}\right)-\phi(x)\right|^{2} \\
& +a L^{d-2} \sum_{y \in \Omega^{(k+1)}}\left|L^{-d} \sum_{x \in B(y)} U\left(A\left(\Gamma_{y, x}\right)\right) \phi(x)\right|^{2}-O\left(e^{2-\delta}\right) \sum_{x \in \Omega^{(k)}}|\phi(x)|^{2} .
\end{aligned}
$$

Now we use again the method we have applied so many times: in the expression on the right hand side above we separate the blocks by Neumann boundary conditions, in each block we decompose $A=A_{0}+A^{\prime}$ into a constant field $A_{0}$ and small field $A^{\prime}$ and we expand with respect to $A^{\prime}$ getting the same expression with $A_{0}$ instead of $A$ and a bigger constant in the last sum. Next we "gauge away" the constant field, and we obtain the Laplace operator with Neumann boundary conditions for each block plus the projection operator on constant functions. This sum is bounded from below by a positive constant (more precisely by $\left.\frac{1}{2} \gamma_{0} \min \left\{\pi^{2} L^{-2}, a L^{-2}\right\}\right)$, thus we have

$$
\left\langle\phi,\left(\Delta^{(k)}(\Omega, A)+a L^{-2} P(A)\right) \phi\right\rangle \geqq \gamma_{0}^{\prime}\langle\phi, \phi\rangle-O\left(e^{2-\delta}\right)\langle\phi, \phi\rangle \geqq \gamma_{0}^{\prime \prime}\langle\phi, \phi\rangle .
$$

for $e$ sufficiently small and we get the lower bound. Finally Corollary 2.3 implies that the considered operator is short-ranged in the sense that for some $\delta_{0}>0$

$$
\left|\left(\Delta^{(k)}(\Omega, A)+a L^{-2} P(A)\right)\left(x, x^{\prime}\right)\right| \leqq c_{0} e^{-\delta_{0}\left|x-x^{\prime}\right|}, \quad x, x^{\prime} \in \Omega^{(k)},
$$


and a change of the domain $\Omega$ implies a change of the operator which can be estimated in the following way

$$
\begin{gathered}
\left|\left(\Delta^{(k)}(\Omega, A)-\Delta^{(k)}\left(\Omega_{0}, A\right)\right)\left(x, x^{\prime}\right)\right| \leqq c_{0} e^{-\delta_{0}\left(\left|x-x^{\prime}\right|+\operatorname{dist}\left(x, \Omega^{(k) c}\right)+\operatorname{dist}\left(x^{\prime}, \Omega^{(k) c}\right)\right)}, \\
\Omega \subset \Omega_{0}, \quad x, x^{\prime} \in \Omega^{(k)} .
\end{gathered}
$$

From these properties it follows that Proposition I.2.3 is a consequence of the following

Theorem. Let $\Omega \subset Z^{d}$ and let $A$ be a symmetric operator defined on the space $L^{2}(\Omega)$ of functions $\phi: \Omega \rightarrow R^{N}$ and satisfying the following condition: there exist positive constants $\gamma_{0}, c_{0}, \delta_{0}$ such that

$$
A \geqq \gamma_{0} I, \quad\left|A\left(x, x^{\prime}\right)\right| \leqq c_{0} e^{-\delta_{0}\left|x-x^{\prime}\right|}, \quad x, x^{\prime} \in \Omega .
$$

Then there exist positive constants $c_{1}, \delta_{1}$ such that for arbitrary $\Lambda \subset \Omega$ and for $C_{\Lambda}=A_{\Lambda}^{-1}, A_{\Lambda}$ is an operator defined on $L^{2}(\Lambda)$ by $A_{\Lambda}=\Lambda A \Lambda$. We have

$$
\begin{gathered}
\left|C_{\Lambda}\left(x, x^{\prime}\right)\right| \leqq c_{1} e^{-\delta_{1}\left|x-x^{\prime}\right|}, \quad x, x^{\prime} \in \Lambda, \\
\left|\delta C_{\Lambda}\left(x, x^{\prime}\right)\right| \leqq c_{1} e^{-\delta_{1}\left(\left|x-x^{\prime}\right|+\operatorname{dist}\left(x, \Lambda^{c}\right)+\operatorname{dist}\left(x^{\prime}, \Lambda^{c}\right)\right)}, \quad \delta C_{\Lambda}=C_{\Lambda}-C_{\Omega} .
\end{gathered}
$$

If we perturb the operator $A$ by an operator B such that the condition (5.6) is satisfied for $A+B$, and additionally $B$ has the property

$$
\left|B\left(x, x^{\prime}\right)\right| \leqq c_{0} e^{-\delta_{0}\left(\left|x-x^{\prime}\right|+\operatorname{dist}\left(x, \Omega^{c}\right)+\operatorname{dist}\left(x^{\prime}, \Omega^{c}\right)\right)}, \quad x, x^{\prime} \in \Omega
$$

then we have also

$$
\left|A_{\Lambda}^{-1}\left(x, x^{\prime}\right)-(A+B)_{\Lambda}^{-1}\left(x, x^{\prime}\right)\right| \leqq c_{1} e^{-\delta_{1}\left(\left|x-x^{\prime}\right|+\operatorname{dist}\left(x, \Omega^{c}\right)+\operatorname{dist}\left(x^{\prime}, \Omega^{c}\right)\right)}, \quad x, x^{\prime} \in \Lambda .
$$

The rest of the section will be devoted to a

Proof of the Theorem. The proof goes along the lines of the proof of Proposition I.2.1. We start with a construction of a "generalized random walk" representation. We define the sets

$$
\square_{j}=\Lambda \cap\left\{x \in Z^{d}:-M \leqq x_{\mu}-M j_{\mu}<M, \mu=1, \ldots, d\right\}, \quad j \in Z^{d},
$$

and we introduce the same partition of unity $h_{j}$ as before. In the sequel we restrict ourselves to these $j$ 's for which $\square_{j} \neq \phi$. Let us define

$$
C_{j}=C_{\square_{j}}, \quad C=\sum_{j} h_{j} C_{j} h_{j} .
$$

We will show again that $C$ is a very good approximation of $C_{A}$ in the sense that $A_{\Lambda} C$ is almost an identity. We have

$$
\begin{aligned}
A_{\Lambda} C & =\sum_{j} \Lambda A h_{j} C_{j} h_{j}=\sum_{j} \square_{j} A h_{j} C_{j} h_{j}+\sum_{j, j^{\prime}, j \neq j^{\prime}}\left(1-\square_{j}\right) h_{j^{\prime}}^{2} A h_{j} C_{j} h_{j} \\
& =\sum_{j} h_{j} A_{\square_{j}} C_{j} h_{j}+\sum_{j} \square_{j}\left[A, h_{j}\right] C_{j} h_{j}+\sum_{j, j^{\prime}, j \neq j^{\prime}}\left(1-\square_{j^{\prime}}\right) h_{j}^{2} A h_{j^{\prime}} C_{j^{\prime}} h_{j^{\prime}} \\
& =I-R,
\end{aligned}
$$


where

$$
R=\sum_{j, j^{\prime}} R_{j, j^{\prime}} C_{j^{\prime}} h_{j^{\prime}}, \quad R_{j, j}=-\square_{j}\left[A, h_{j}\right] \square_{j}, \quad R_{j, j^{\prime}}=-\left(1-\square_{j^{\prime}}\right) h_{j}^{2} A h_{j^{\prime}} \quad \text { for } \quad j \neq j^{\prime} .
$$

It is natural to interpret $R_{j, j^{\prime}}$ as an operator $R_{j, j^{\prime}}: L^{2}\left(\square_{j^{\prime}}\right) \rightarrow L^{2}\left(\square_{j}\right)$, similarly $R_{j, j^{\prime}} C_{j^{\prime}}, h_{j^{\prime}}$. We have

$$
\begin{aligned}
\left|R_{j, j}\left(x, x^{\prime}\right)\right| & =\left|A\left(x, x^{\prime}\right)\left(h_{j}\left(x^{\prime}\right)-h_{j}(x)\right)\right| \leqq c_{0} e^{-\delta_{0}\left|x-x^{\prime}\right|} O(1) \frac{\left|x-x^{\prime}\right|}{M}, \quad x, x^{\prime} \in \square_{j}, \\
\left|R_{j, j^{\prime}}\left(x, x^{\prime}\right)\right| & =\left|\left(1-\square_{j^{\prime}}\right)(x) h_{j}^{2}(x) A\left(x, x^{\prime}\right) h_{j^{\prime}}\left(x^{\prime}\right)\right| \leqq\left(1-\square_{j},(x)\right) c_{0} e^{-\delta_{0}\left|x-x^{\prime}\right|} h_{j^{\prime}}\left(x^{\prime}\right) \\
& \leqq c_{0} e^{-\frac{1}{2} \delta_{0}\left|x-x^{\prime}\right|} e^{-\frac{1}{6} \delta_{0} M} \quad \text { for } \quad x \in \square_{j}, \quad x^{\prime} \in \square_{j^{\prime}},
\end{aligned}
$$

and these estimates imply that the norms of the operators $R_{j, j^{\prime}}$ have bounds

$$
\left\|R_{j, j^{\prime}}\right\| \leqq \alpha e^{-\delta_{2}\left|j-j^{\prime}\right|}
$$

for $\alpha$ depending on $M$ and arbitrarily small if $M$ is sufficiently large, $\delta_{2}$ depending on $\delta_{0}$, e.g. $\delta_{2}=\frac{1}{4} \delta_{0}$. From these bounds it follows that

$$
\|R\| \leqq \max \left\{\sup _{j} \sum_{j^{\prime}}\left\|R_{j, j^{\prime}}\right\|, \sup _{j^{\prime}} \sum_{j}\left\|R_{j, j^{\prime}}\right\|\right\} \gamma_{0}^{-1} \leqq \alpha \gamma_{0}^{-1} \sum_{j \in Z^{d}} e^{-\delta_{2}|j|}
$$

is small for $M$ large and

$$
C_{\Lambda}=C(I-R)^{-1}=\sum_{n=0}^{\infty} C R^{n}
$$

which can be given a random walk form:

$$
C_{A}=\sum_{\omega} h_{\omega_{0}} C_{\omega_{0}} h_{\omega_{0}} R_{\omega_{1}, \omega_{2}} C_{\omega_{2}} h_{\omega_{2}} \cdot \ldots \cdot R_{\omega_{2 n-1}, \omega_{2 n}} C_{\omega_{2 n}} h_{\omega_{2 n}}
$$

$\omega=\left(\omega_{0}, \omega_{1}, \ldots, \omega_{2 n}\right), \omega_{i}$ are arbitrary indices $j$, but satisfying the restrictions

$$
\max _{\mu=1, \ldots, d}\left|\omega_{2 i, \mu}-\omega_{2 i+1, \mu}\right| \leqq 1 \text { for } i=0,1, \ldots, n-1, \quad n \geqq 0 .
$$

Let us now prove (5.7). We have

$$
\begin{aligned}
& \left|C_{\Lambda}\left(x, x^{\prime}\right)\right| \sum_{\omega: x \in \square \omega_{0}, x^{\prime} \in \square \omega_{2 n}}\left|\left\langle\delta_{x}, h_{\omega_{0}} C_{\omega_{0}} \ldots R_{\omega_{2 n-1}, \omega_{2 n}} C_{\omega_{2 n}} h_{\omega_{2 n}} \delta_{x^{\prime}}\right\rangle\right| \\
& \leqq \sum_{\omega}^{\prime} \gamma_{0}^{-(n+1)} \alpha^{n} e^{-\delta_{2}\left|\omega_{1}-\omega_{2}\right|} \ldots \cdot e^{-\delta_{2}\left|\omega_{2 n-1}-\omega_{2 n}\right|} \\
& \leqq \\
& \quad \sum_{j, j^{\prime}: x \in \square, j, x^{\prime} \in \square}\left[\sum_{j^{\prime}}^{\infty} \gamma_{0}^{-(n+1)} \alpha^{n}\left(e^{d \delta_{2}}\right)^{n}\right. \\
& \left.\quad \cdot \sum_{j_{1}, \ldots, j_{2 n-1} \in Z^{d}} e^{-\delta_{2}\left|j-j_{1}\right|} \cdot \ldots \cdot e^{-\delta_{2}\left|j_{2 n-1}-j^{\prime}\right|}+\gamma_{0}^{-1} e^{d \delta_{2}} e^{-\delta_{2}\left|j-j^{\prime}\right|}\right] .
\end{aligned}
$$

To investigate the sums above, it is convenient to take the absolute value $|j|$ defined by $|j|=\sum_{\mu=1}\left|j_{\mu}\right|$; then the sum over $j$ 's factorizes into sums over components. If we define $g_{1}(j)=e^{-\delta_{2}|j|}, j \in Z$, then the sum over $j$ 's in (5.18) can be written as $\prod_{\mu=1}^{d} g_{2 n}\left(j_{\mu}-j_{\mu}^{\prime}\right), g_{2 n}=g_{1} * \ldots * g_{1}$ is a convolution of $2 n$ functions $g_{1}$. The Fourier transform of $g_{1}$ is equal to 


$$
\sum_{j \in Z} e^{-i p j} e^{-\delta_{2}|j|}=\left(1-e^{-i p-\delta_{2}}\right)^{-1}+\left(1-e^{i p-\delta_{2}}\right)^{-1}-1=\frac{1-e^{-2 \delta_{2}}}{\left(1-e^{-i p-\delta_{2}}\right)\left(1-e^{i p-\delta_{2}}\right)},
$$

so we have

$$
g_{n}(j)=\frac{1}{2 \pi} \int_{-\pi}^{\pi} d p e^{i j p} \frac{\left(1-e^{-2 \delta_{2}}\right)^{n}}{\left(1-e^{-i p} e^{-\delta_{2}}\right)^{n}\left(1-e^{i p} e^{-\delta_{2}}\right)^{n}}, \quad g_{n}(j)=g_{n}(|j|),
$$

and from this we obtain

$$
\left|g_{n}(j)\right| \leqq c_{2}^{n} e^{-\frac{1}{2} \delta_{2}|j|}, \text { for some } c_{2} .
$$

Finally we fix $M$ such that $\gamma_{0}^{-1} \alpha e^{d \delta_{2}} c_{2}^{2 d}<1$, and we get

$$
\begin{aligned}
\left|C_{\Lambda}\left(x, x^{\prime}\right)\right| & \leqq \sum_{j, j^{\prime}: x \in \square_{J}, x^{\prime} \in \square_{J^{\prime}}} \gamma_{0}^{-1} e^{d \delta_{2}} \frac{1}{1-\gamma_{0}^{-1} e^{d \delta_{2}} c_{2}^{2 d} \alpha} e^{-\frac{1}{2} \delta_{2}\left|j-j^{\prime}\right|} \\
& \leqq c_{1} e^{-\frac{1}{2} \delta_{2} M^{-1}\left|x-x^{\prime}\right|} .
\end{aligned}
$$

This inequality implies (5.7) with $\delta_{1}=\frac{1}{2} \delta_{2} M^{-1}$. The inequality (5.8) is proved using the representations (5.17) for both operators $C_{A}, C_{\Omega}$ and properly taking into account cancellations of some terms, exactly as in the proof of bounds for $\delta G_{k}\left(\Omega, \Omega_{0}, A\right)$ in Sect. 2 .

We define $\square_{j}^{\prime}$ by the equality (5.11) with $\Omega$ instead of $\Lambda$, and $C_{j}^{\prime}=C_{\square_{j}^{\prime}}$. If $\square_{j}^{\prime}$ is disjoint with the complement $\Lambda^{c}$ of $\Lambda$ in $\Omega$, then $\square_{j}^{\prime}=\square_{j}$ and $C_{j}^{\prime}=C_{j}$. Similarly if both $\square_{j}^{\prime}, \square_{j^{\prime}}^{\prime}$ are disjoint with $\Lambda^{c}$, then $R_{j, j^{\prime}}^{\prime}=R_{j, j^{\prime}}$. Let us take a difference of the representations (5.17) for $C_{A}$ and $C_{\Omega}$. In this difference all terms corresponding to walks $\omega$ with $\square_{\omega_{i}}^{\prime}$ disjoint with $\Lambda^{c}$ are canceled, and we have

$$
\begin{aligned}
& \delta C_{\Lambda}\left(x, x^{\prime}\right)
\end{aligned}
$$

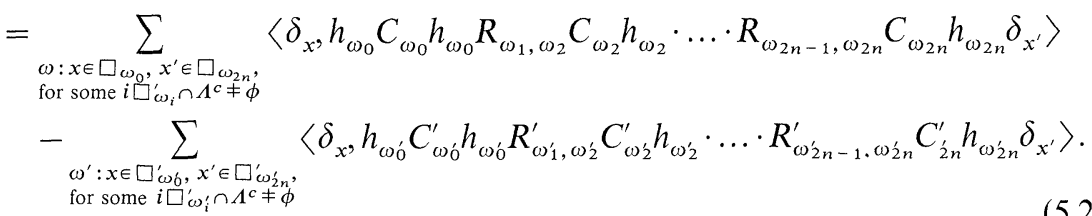

The range of $\omega_{i}^{\prime}$ in the second sum might be larger because $\Omega \supset \Lambda$. Estimating (5.22) in the same way as in (5.18) and (5.21), we get

$$
\left|\delta C_{\Lambda}\left(x, x^{\prime}\right)\right| \leqq c_{1}^{\prime} e^{-\frac{1}{3} \delta_{2} M^{-1}\left(\operatorname{dist}\left(x, \Lambda^{c}\right)+\operatorname{dist}\left(x^{\prime}, \Lambda^{c}\right)+\left|x-x^{\prime}\right|\right)} .
$$

A proof of the last inequality (5.10) is very similar, although a little bit more awkward. We form the representations (5.17) for $A_{\Lambda}^{-1}$ and $(A+B)_{\Lambda}^{-1}$, and we take their difference:

$$
\begin{aligned}
A_{\Lambda}^{-1}-(A+B)_{\Lambda}^{-1}= & \sum_{\omega}\left[h_{\omega_{0}} C(A)_{\omega_{0}} h_{\omega_{0}} R(A)_{\omega_{1}, \omega_{2}}\right. \\
& \cdot C(A)_{\omega_{2}} h_{\omega_{2}} \cdot \ldots \cdot R(A)_{\omega_{2 n-1}, \omega_{2 n}} C(A)_{\omega_{2 n}} h_{\omega_{2 n}} \\
& -h_{\omega_{0}} C(A+B)_{\omega_{0}} h_{\omega_{0}} R(A+B)_{\omega_{1}, \omega_{2}} C(A+B)_{\omega_{2}} h_{\omega_{2}} \cdot \ldots \\
& \left.\cdot R(A+B)_{\omega_{2 n-1}, \omega_{2 n}} C(A+B)_{\omega_{2 n}} h_{\omega_{2 n}}\right] \\
= & \sum_{\omega}\left[h _ { \omega _ { 0 } } \left(C(A)_{\omega_{0}}-C(A+B)_{\omega_{0}} h_{\omega_{0}} R(A)_{\omega_{1}, \omega_{2}} C(A)_{\omega_{2}} h_{\omega_{2}} \cdot \ldots\right.\right. \\
& +h_{\omega_{0}} C(A+B)_{\omega_{0}} h_{\omega_{0}}\left(R(A)_{\omega_{1}, \omega_{2}}-R(A+B)_{\omega_{1}, \omega_{2}}\right) \\
& \left.\cdot C(A)_{\omega_{2}} h_{\omega_{2}} \cdot \ldots+\ldots\right] .
\end{aligned}
$$


From the definition (5.14) of the operators $R_{j, j^{\prime}}$ we have

$$
R(A)_{j, j^{\prime}}-R(A+B)_{j, j^{\prime}}=-R(B)_{j, j^{\prime}},
$$

and from the condition (5.9), we get the estimate

$$
\left\|R(B)_{j, j^{\prime}}\right\| \leqq \alpha e^{-\delta_{2}\left|j-j^{\prime}\right|} e^{-\delta_{0}\left(\operatorname{dist}\left(\square_{j}, \Omega^{c}\right)+\operatorname{dist}\left(\square_{j^{\prime}}, \Omega^{c}\right)\right)} .
$$

Further we have

$$
C(A)_{j}-C(A+B)_{j}=A_{\square_{j}}^{-1}-(A+B)_{\square_{j}}^{-1}=A_{\square_{j}^{-1}}^{-1} B_{\square_{j}}(A+B)_{\square_{j}}^{-1},
$$

and

$$
\left\|C(A)_{j}-C(A+B)_{j}\right\| \leqq \gamma_{0}^{-2} c_{0}^{\prime} e^{-2 \delta_{0} \operatorname{dist}\left(\square_{J}, \Omega^{c}\right)} .
$$

The equality (5.24) and the above estimates imply

$$
\begin{aligned}
& \left|A_{\Lambda}^{-1}\left(x, x^{\prime}\right)-(A+B)_{\Lambda}^{-1}\left(x, x^{\prime}\right)\right| \\
& \leqq \sum_{\omega: x \in \square \omega_{0}, x^{\prime} \in \square \omega_{2 n}} c_{0}^{\prime} \gamma_{0}^{-(n+2)} \alpha^{n}\left(e^{d \delta_{2}}\right)^{n} e^{-\delta_{2}\left|\omega_{0}-\omega_{1}\right|} \ldots \cdot e^{-\delta_{2}\left|\omega_{2 n-1}-\omega_{2 n}\right|} \\
& \cdot\left(\sum_{i=1}^{n} e^{-2 \delta_{0} \operatorname{dist}\left(\square \omega_{2 i}, \Omega^{c}\right)}+\sum_{i=1}^{n} e^{-\delta_{0}\left(\operatorname{dist}\left(\square \omega_{2 t-1}, \Omega^{c}\right)+\operatorname{dist}\left(\square \omega_{22}, \Omega^{c}\right)\right)}\right) \\
& \leqq e^{-\frac{1}{3} \delta_{2} M^{-1}\left(\operatorname{dist}\left(x, \Omega^{c}\right)+\operatorname{dist}\left(x^{\prime}, \Omega^{c}\right)\right)} \gamma_{0}^{-2} c_{0}^{\prime} e^{2 d \delta_{2}} \frac{1}{1-\gamma_{0}^{-1} 4 e^{d \delta_{2}} c_{2}^{2 d} \alpha} e^{-\frac{1}{3} \delta_{2} M^{-1}\left|x-x^{\prime}\right|} .
\end{aligned}
$$

Thus Inequality (5.10) is proved. The constants $\delta_{1}, c_{1}$ are functions of $\delta_{0}, \gamma_{0}, c_{0}$, and from the above proof we can get more precise estimates for them.

\section{References}

1. Bałaban, T.: (Higgs) $)_{2,3}$ quantum fields in a finite volume. I. A lower bound. Commun. Math. Phys. 85, 603-636 (1982)

2. Bałaban, T.: (Higgs $)_{2,3}$ quantum fields in a finite volume. II. An upper bound. Commun. Math. Phys. 86, 555-594 (1982)

3. Bałaban, T.: (Higgs) ${ }_{2,3}$ quantum fields in a finite volume. III. Renormalization. Commun. Math. Phys. 88, 411-445 (1983) (or Harvard preprints HUTMP 82/B116, B117, B119)

4. Brydges, D.C., Federbush, P.: A lower bound for the mass of a random Gaussian lattice. Commun. Math. Phys. 62, 79-82 (1978)

5. Brydges, D.C., Fröhlich, J., Seiler, E.: On the construction of quantized gauge fields. I. General results. Ann. Phys. 121, 227-284 (1979)

6. Dunford, N., Schwartz, J. : Linear operators Part I, Chap. VI. 10. Interscience Publishers, Inc. New York : 1958

Communicated by A. Jaffe

Received February 14, 1983 
